GSA Data Repository 2016189

\title{
SUPPLEMENTARY METHODOLOGY
}

1.1 Sampling: This study is based on the compositional analysis of 128 sediment samples collected in the Galápagos Archipelago (GAL), during cruises in June of 2012 and March of 2013, through the use of SCUBA and Van Veen grab, at sites spanning the geographic and oceanographic extent of the GAL. Water depths ranged from 1-40m depths. All GAL samples were dried for 30 hours in oven at $56^{\circ} \mathrm{C}$. GOC The Gulf of California (GOC) analyses in this study are based on the results of raw sediment count data collected and analyzed by Halfar et al. (2006). 128 surface SCUBA and Van Veen grab samples were collected from shallow (subtidal) to $40 \mathrm{~m}$ along an $800 \mathrm{~km}$ latitudinal transect along the western shelf of the GOC (Fig. 1C).

1.2 Component Analysis: All samples were wet sieved to separate out the sand fraction $(63 \mu \mathrm{m}$ to $2 \mathrm{~mm}$ fraction from the gravel fraction $(>2 \mathrm{~mm})$ and $\mathrm{mud} / \mathrm{silt}$ fraction $(<63 \mu \mathrm{m})$. Thin sections were made from sand fraction. A 300 point-per-sample count of carbonate (siliciclastics removed) grains was performed on the sand fraction $(63 \mu \mathrm{m}-2 \mathrm{~mm})$ of thin sections, using an automated slide stage and petrographic scope under cross-polarized 40X magnification. Samples were counted according to numbers of molluscs, corals, echinoderms, barnacles, serpulids, coralline algae, bryozoans, crustaceans, benthic foraminifera, planktonic forminifera, and unidentified grains in each counted sample.

1.3 Oceanographic Data Collection and Analysis: While in-situ chlorophyll-a (Chl-a), sea surface temperature (SST), and sea surface salinity (SSS) data of a number of the collection sites in question do exist, time series are generally too sporadic or short ( $<5$ years) to be used to produce any long-term oceanographic averages. As a result of this in-situ data limitation, Moderate Resolution Imaging Spectroradiometer (MODIS) Chl-a (4km, 8-day composite from 4 July 2002 - 10 November 2014) and SST (4 km monthly data from July 2002 - November 2014) were collected for individual Galápagos collection sites using NASA, Giovanni data (http://disc.sci.gsfc.nasa.gov/giovanni). Note: all trophic 
definitions used in the manuscript are based on Chl-a categorizations of Hallock (2001) and SST categorizations of Betzler et al. (1997).

Average monthly sea surface salinity (SSS) data were compiled using Simple Ocean Data Assimilation (SODA) mean monthly estimates for 1990-2010 using the SODA - POP 2.2.4 Monthly Means data set estimates, calculated for $5 \mathrm{~m}$ depth (http://coastwatch.pfeg.noaa.gov/erddap/griddap/hawaii d90f 20ee c4cb.html).

Values for the GAL $\mathrm{pH}$ were obtained by averaging in-situ $\mathrm{pH}$ means from all collection sites, measured in 2003 (La Niña), 2009 (El Niño) and 2012 (Manzello et al., 2010). Surface pH in the GOC was acquired from $\mathrm{pH}$ data collected by Zirino and colleagues in the late 1990's, along a latitudinal transect of the Gulf (Zirino et al., 1997). These data were selected due to their overlap with the collection sites of Halfar et al. (2006). The full transect data set was divided into 4 latitudinal zones, and averaged to represent the average for each collection zone in Halfar et al. (2006). $\mathrm{pH}$ was chosen over aragonite saturation state $(\Omega)$ due the lack of GOC $\Omega$ data needed for comparison.

1.4 Statistical Analyses: Biogenic count results are based on the percent composition of each biogenic component (siliciclastic material excluded). Euclidean-Ward cluster analyses were performed on the unmodified raw point count results - calculated into percent composition - of each biogenic category, using the 'ape' package in R version 3.2.2 (R Core Team, 2013). The dendrograms were divided into the five most dominant clusters for all fractions (partitioned by R). Samples within each major cluster were binned according to their respective place within the cluster, by numbering them sequentially according to their respective location within their cluster group.

From the raw oceanographic data, several indices were calculated. These indices include the overall Mean, Mean Anomaly, minimum (Min) and maximum (Max) values for Chl-a, SST, and SSS. Mean was calculated from the average of all oceanographic data values at each sample location. MA represents the mean of all oceanographic anomalies, which were calculated as the overall mean, minus the 
mean monthly values. Min and Max were calculated from the raw data values, and represent the overall minimum and maximum values at each sampling location.

Data exploration was performed on all data values, in order to look for the occurrence of outliers and colinearity among the experimental indices (Zuur et al., 2009; Zuur et al., 2010). Although boxplots revealed strong outliers in Chl-a values for the upwelling sites of Isabela (GAL) and Los Angeles (GOC) - which could justify their removal from the data - our investigation into the effects that these ENSOaffected, atypical, upwelling systems have on biogenic sediments, warranted their preservation within the analyses for this study. Essentially, these outlying data represent the extremes of ENSO on these upwelling systems. Additionally, pair-plots of experimental data did not suggest that the relationships between the explanatory variables (Chl-a, SST, SSS, and pH and depth), with the response variable (biogenic cluster analysis results) should not be linear. As a result of this interpretation, a univariate linear regression tree analysis (Zuur et al., 2009; Zuur et al., 2010) was performed for the response and explanatory variables and the resultant tree was trimmed to the best of 4 splits to isolate the key —and most statistically significant — explanatory variables influencing the cluster distribution of the biogenic count data. The response variable for these exploratory analyses were the binned results of each cluster analysis run against the explanatory variables, which were indices calculated from the Chl-a/SST and SSS time series, as well as the $\mathrm{pH}$ averages and individual sample depths. Our work flow is as follows:

1) search species (grain characteristics) -by-site for pattern by cluster analysis

2) show the characteristics of the clusters by pie charts.

3) Now we have groups of samples characterized by a specific distribution of fauna (=grain characteristics)

4) Use regression trees to find which environmental factors best split the data into said groups 


\section{REFERENCES CITED:}

Betzler, C., Brachert, T.C., Nebelsick, J., 1997, The warm temperate carbonate province A review of facies, zonation and delimitations: Courier Forschungsinstitut Senckenberg v. 201, p. 83-99.

Halfar, J., Godinez-Orta, L., Mutti, M., Valdez-Holguin, J. E., and Borges, J. M., 2006, Carbonates calibrated against oceanographic parameters along a latitudinal transect in the Gulf of California, Mexico: Sedimentology, v. 53, no. 2, p. 297-320.

Manzello, D. P., 2010, Ocean acidification hot spots: Spatiotemporal dynamics of the seawater CO2 system of eastern Pacific coral reefs: Limnol. Oceanogr, v.55, p. 239-248.

R Core Team (2013). R: A language and environment for statistical computing. R Foundation for Statistical Computing, Vienna, Austria.URL http://www.R-project.org/.

Rayner, N. A., Parker, D. E., Horton, E. B., Folland, C. K., Alexander, L. V., Rowell, D. P., Kent, E. C., Kaplan, A., Global analyses of sea surface temperature, sea ice, and night marine air temperature since the late nineteenth century: J. Geophys. Res, v. 108 (D14), p. 4407

Zirino, A., Hérnandez-Ayón., Fuhrmann, R.A., Álvarez-Borrego, S., Gaxiola-Castro, G., Lara-Lara, J.R, and Bernstein, R.L.,1997, Estimate of surface PCO, in the Gulf of California from underway pH measurements and satellite imagery: : Ciencias Marinas, v. 23, no. 1.

Zuur, A., Ieno, E. N., Walker, N., Saveliev, A. A., \& Smith, G. M. , 2009, Mixed effects models and extensions in ecology with R, Springer Science \& Business Media.

Zuur, A. F., Ieno, E. N., and Elphick, C. S., 2010, A protocol for data exploration to avoid common statistical problems: Methods in Ecology and Evolution, v. 1, no. 1, p. 3-14. 


\section{SUPPLEMENTARY FIGURE CAPTIONS}

Figure DR1. Maps detailing geographic distribution of biogenic categories within point counts of sediment samples analyzed from GAL (A-H) and GOC (I-P). Count data is presented as bubbles, detailing percent component of each biogenic category at each collection site. Gray keys within each map show relative bubble percentages for comparison. Note: Maps for GAL and GOC use different bubble size-scale.

Figure DR2. Cluster diagrams, showing the 5 major sedimentological clusters for the Galápagos (A; I-V) and the Gulf of California (B; A-E), with bar charts detailing the fractions of major biogenic categories within each cluster group. 


\section{SUPPLEMENTARY TABLE CAPTIONS}

Table DR1. Calculated oceanographic parameters used in exploratory analyses for GAL (A) and GOC (B). Column names are individual collection sites within each study region. Min and Max represent overall minimum and maximum values for their respective data sets. Mean Anomaly represents the mean of all anomaly values, which were calculated by subtracting overall mean from individual data values

Table DR2. Point count percentages by biogenic component, cluster analysis bin number, depth, and sample location (decimal degree). Site codes: D: Darwin; B: Baltra; SF: San Cristóbal; E: Española; F: Floreana; I: Isabela.

Table DR3. Details of the average percentages of biogenic groups within each group (I-V for Galápagos and A_E for Gulf of California) of the cluster analysis. 


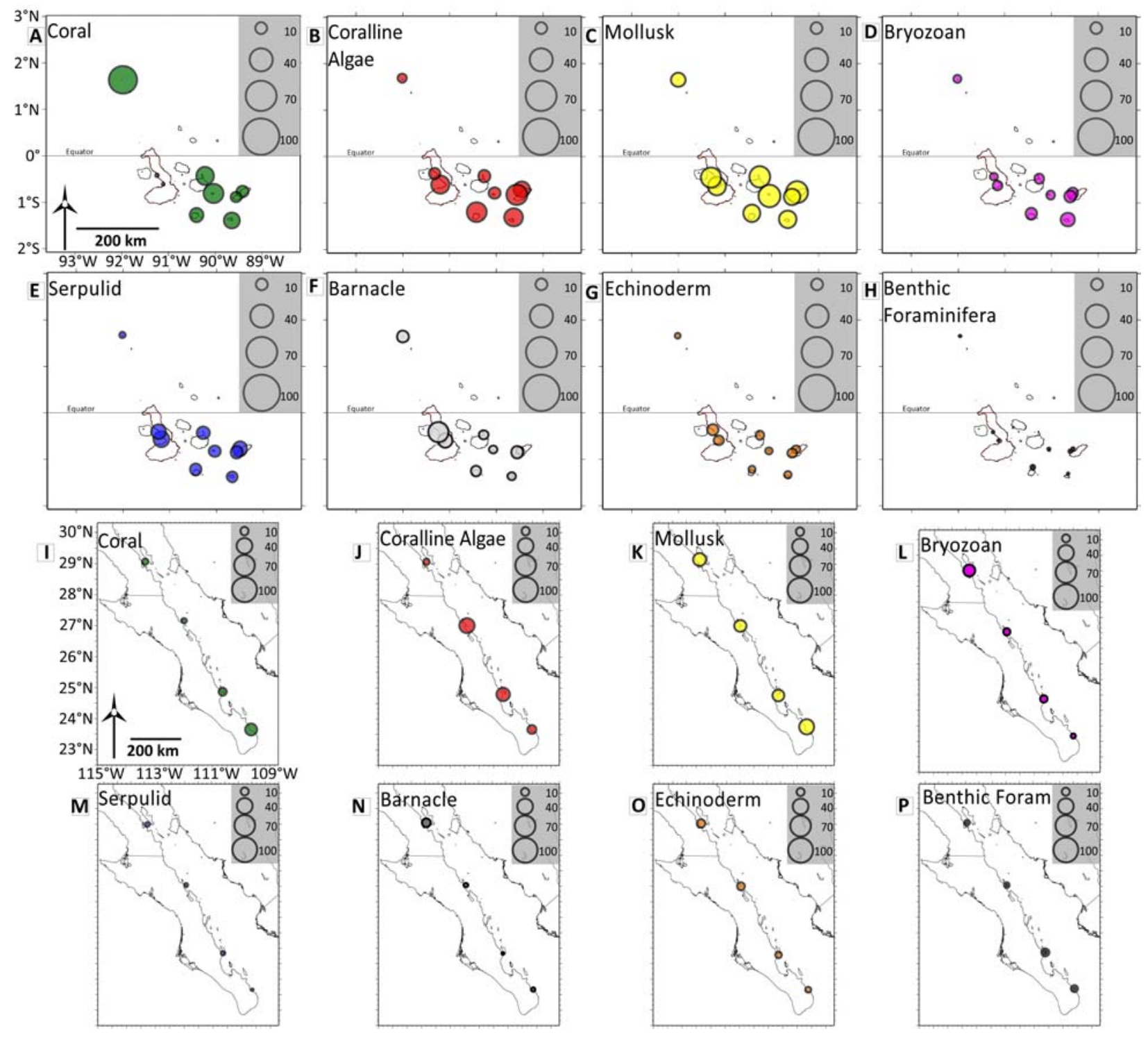

Figure DR1 
A

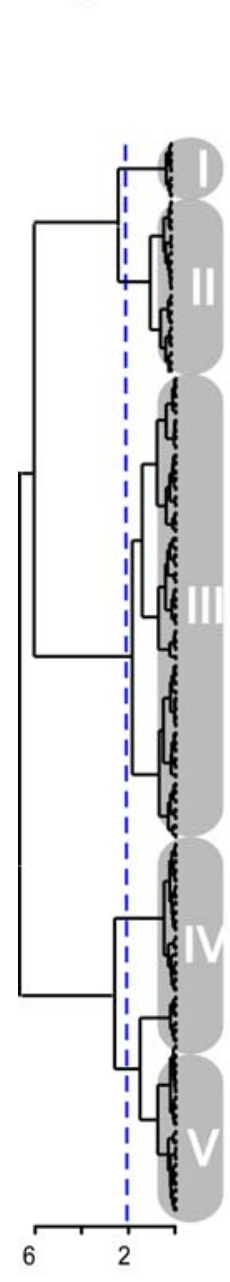

Distance (x100)
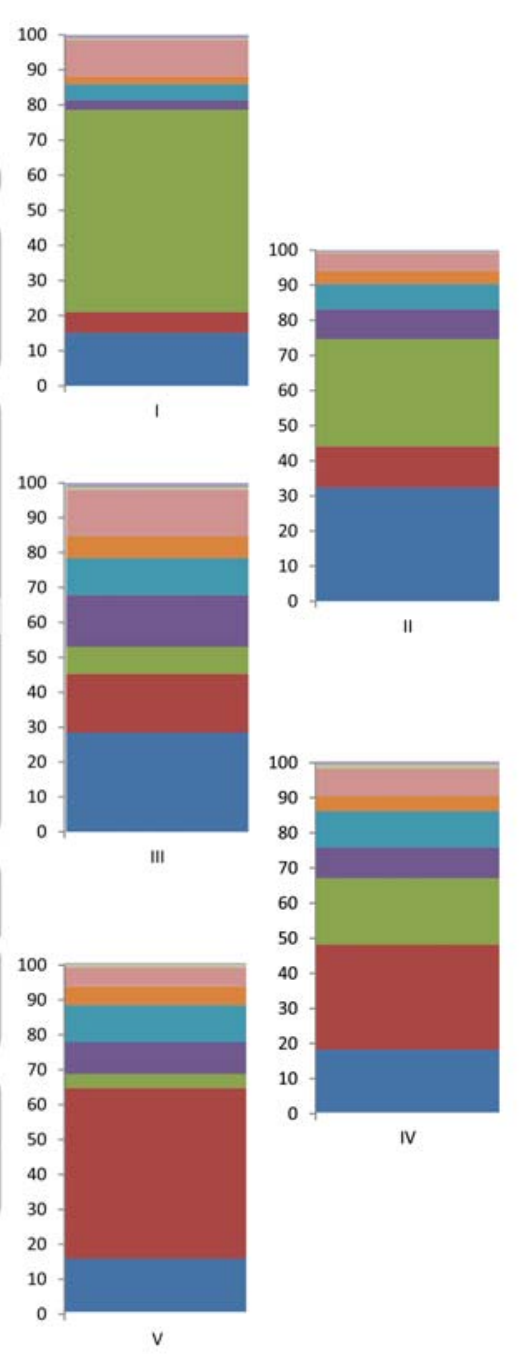

B

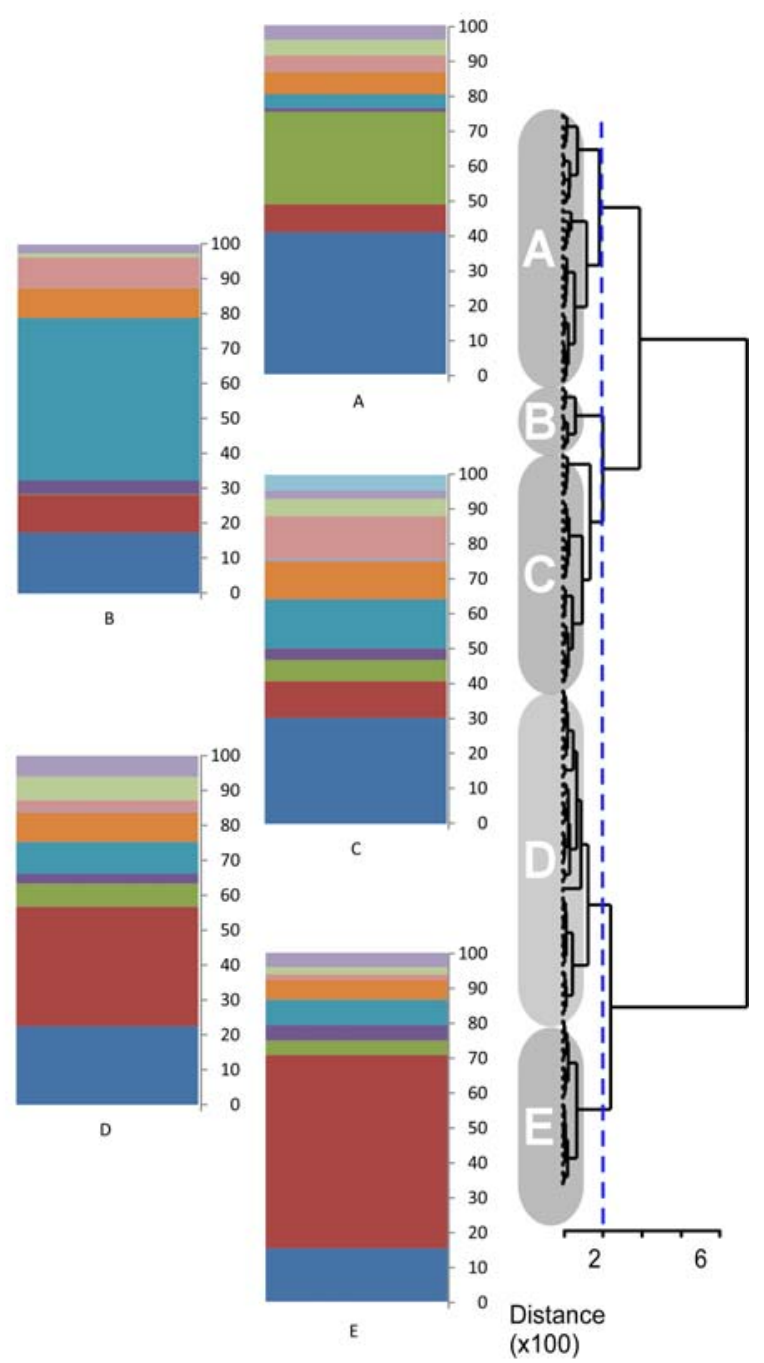

Other

Benthic Foram

Barnacle

Sponge

Echinoderm

Bryozoan

口Serpulid

coral

- Coralline Algae

Mollusk

Figure DR2

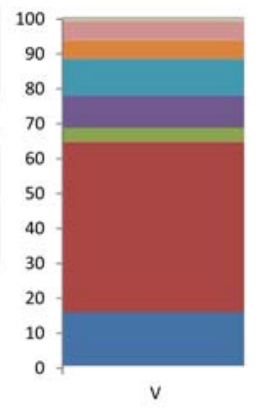




\section{Table DR1}

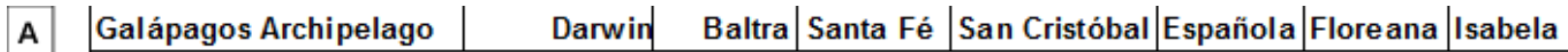

\begin{tabular}{|lrrrrrrr|}
\hline Chl-a $\left(\mathbf{m g} / \mathbf{m}^{\wedge} \mathbf{3}\right)$ & 0.21 & 0.36 & 0.35 & 0.49 & 0.30 & 0.33 & 2.01 \\
Mean & 0.06 & 0.10 & 0.14 & 0.15 & 0.13 & 0.15 & 0.17 \\
Min & 0.60 & 2.27 & 1.36 & 2.74 & 0.97 & 0.92 & 20.73 \\
Max & 0.06 & 0.12 & 0.10 & 0.21 & 0.07 & 0.09 & 1.48 \\
Mean Anomaly & & & & & & & \\
\hline SST ( $\left.{ }^{\circ} \mathbf{C}\right)$ & 25.56 & 23.80 & 23.48 & 23.44 & 23.53 & 23.41 & 22.96 \\
Mean & 22.25 & 19.44 & 18.04 & 19.56 & 18.91 & 18.81 & 19.06 \\
Min & 29.62 & 28.43 & 27.88 & 28.46 & 28.32 & 27.99 & 27.85 \\
Max & 1.44 & 1.83 & 1.91 & 1.78 & 1.93 & 2.02 & 2.17 \\
Mean Anomaly & & & & & & & \\
\hline SSS (PSU) & 33.54 & 34.11 & 34.25 & 34.24 & 34.40 & 34.25 & 34.23 \\
Mean & 32.27 & 32.89 & 33.04 & 33.02 & 33.18 & 33.07 & 32.84 \\
Min & 34.78 & 35.09 & 35.18 & 35.14 & 35.22 & 35.20 & 35.36 \\
Max & 0.38 & 0.39 & 0.37 & 0.37 & 0.33 & 0.36 & 0.40 \\
Mean Anomaly & & & & & & & \\
\hline pH (full scale) & 8.07 & 7.94 & 7.97 & 7.90 & 8.03 & 7.91 & 7.88 \\
\hline Average pH & & & & & & &
\end{tabular}

\begin{tabular}{|c|c|c|c|c|}
\hline Gulf of California & Cabo Pulmo & San José & Punta Chivato & Los Ángeles \\
\hline \multicolumn{5}{|l|}{ Chl-a (mg/m^3) } \\
\hline Mean & 0.49 & 0.80 & 1.12 & 2.63 \\
\hline Min & 0.09 & 0.12 & 0.16 & 0.48 \\
\hline Max & 4.48 & 6.68 & 9.10 & 19.61 \\
\hline Mean Anomaly & 0.34 & 0.47 & 0.69 & 1.45 \\
\hline \multicolumn{5}{|l|}{ SST $\left({ }^{\circ} \mathrm{C}\right)$} \\
\hline Mean & 26.08 & 25.40 & 24.45 & 22.16 \\
\hline Min & 19.01 & 18.66 & 16.38 & 14.53 \\
\hline Max & 31.73 & 31.69 & 31.59 & 30.02 \\
\hline Mean Anomaly & 3.12 & 3.20 & 4.07 & 4.55 \\
\hline \multicolumn{5}{|l|}{ SSS (PSU) } \\
\hline Mean & 35.11 & 35.49 & 35.80 & 35.79 \\
\hline Min & 33.76 & 34.52 & 35.17 & 35.38 \\
\hline Max & 36.40 & 36.36 & 36.42 & 36.21 \\
\hline Mean Anomaly & 0.33 & 0.24 & 0.19 & 0.11 \\
\hline \multicolumn{5}{|l|}{ pH (full scale) } \\
\hline Average $\mathrm{pH}$ & 8.09 & 8.09 & 8.07 & 7.96 \\
\hline
\end{tabular}


Table DR2.

Sample

(62 $\mu \mathrm{m}-2 \mathrm{~mm})$

Cluster Bin Degree Dec Degree Dec Depth (m) Mollusc (\%)

D_A_43

D_B_43

DRS

DS_2A

DS_2B

DS_7

DS_9

G_B-1

G_B-2

G_B-3

G_B-5

G_B-6

G_SF_N-7

G_SF_N-8

G_SF_N-9

G_SF_N10

G_SF_N-11

G_SF_N-12

G_SF_N-13

G_SC_BM-14

G_SC_BM-15

G_SC_BM-16

G_SC_BM-17

G_SC_BM-18

G_SC_BM-19

G_SC_BM-20

G_SC_BM-21

G_SC_BM-22

403

1.67356
1.67356
1.67356
1.67356
1.67356
1.67356
1.67356
-0.48867

$-91.99213$

13.1

16.3

402

$-91.99213$

13.1

16.5

405

$-91.99213$

12.5

12.7

406

$-91.99213$

13.7

16.7

407

$-91.99213$

13.7

20.7

404

$-91.99213$

13.7

13.8

$-91.99213$

13.7

9.1

510

$-90.27058$

33.3

$508 \quad-0.48867$

$-90.27058$

4.3

35.4

$-0.48867$

$-90.27058$

3.7

23.5

501

$-0.48902$

$-90.3025$

2.7

42.8

505

$-0.48902$

$-90.3025$

32.3

514

$-90.0379$

30.4

511

$-90.0379$

34.8

506

$-90.0391$

31.0

512

$-90.03828$

$-90.03717$

35.7

509

$-90.0359$

37.8

502

$-90.03413$

43.4

503

$-89.54517$

45.4

$-0.80552$

$-89.54517$

36.9

311

$-0.84552$

$-89.54517$

35.2

103

$-0.84552$

$-89.54517$

22.2

312

$-0.84552$

$-89.54517$

31.0

$-0.84552$

$-89.54685$

32.8

320

$-0.84552$

$-89.55108$

39.5

316

$-0.84328$

$-89.55215$

$-0.84242$

$-89.55328$

$-0.8402$

$-89.55567$

G_SC_IL-24

G_SC_IL-25

G_SC_IL-26

G_SC_IL-27

G_SC_IL-28

G_SC_IL-29

G_SC_IL-30

G_SC_IL-31

G_SC_IL-32

G_SC_IL-33

G_SC_IL-35

G_SC_IL-36

G_SC_IL-38

G_SC_IL-39

G_SC_IL-40

G_SC_IL-41

G_SC_IL-42

$-0.85565$

$-89.56108$

$-89.56108$

$-0.85565$

$-89.56108$

$-0.85565$

$-89.56108$

$-89.56108$

$-89.56067$

$-0.85645$

$-0.85517$

$-89.55963$

$-89.55937$

$-89.56012$

$-0.85093$

$-89.56092$

$-89.56883$

$-89.57023$

$-89.57405$

$-89.573$

$-89.56728$

$-89.56588$

37.8

15.0

39.5

20.0

41.5

23.0

42.7

28.0

23.4

6.0

33.6

5.0

27.3

4.0

31.5

$\begin{array}{ll}4.0 & 26.9\end{array}$

$\begin{array}{ll}6.5 & 39.6\end{array}$

$\begin{array}{ll}6.5 & 10.7\end{array}$

16.0

10.9

20.0

40.5

$-0.85063$

$-0.85252$

$-0.85743$

$-0.85858$

$-0.85728$

$-0.85665$

$-89.57373$

26.0

13.0

27.0

20.1

22.0

3.4

26.0

12.4

115

24.0

17.7

116

$-0.85842$

24.0

17.2

7.0

14.3

337

25.0

9.2 


\begin{tabular}{|c|c|c|c|c|c|}
\hline $\begin{array}{l}\text { Sample } \\
(62 \mu \mathrm{m}-2 \mathrm{~mm})\end{array}$ & Cluster Bin & Degree Dec & Degree Dec & Depth (m) & Mollusc (\%) \\
\hline G_SC_IL-43 & 217 & -0.88528 & -89.60787 & 15.0 & 12.5 \\
\hline G_SC_IL-44 & 202 & -0.88625 & -89.60767 & 14.0 & 11.7 \\
\hline G_SC_IL-45 & 218 & -0.88727 & -89.60738 & 11.0 & 13.5 \\
\hline G_SC_IL-46 & 328 & -0.8864 & -89.61037 & 27.0 & 39.4 \\
\hline G_SC_IL-47 & 335 & -0.88463 & -89.60767 & 17.0 & 16.8 \\
\hline G_SC_IL-48 & 211 & -0.88232 & -89.6079 & 30.0 & 16.1 \\
\hline G_SC_IL-50 & 203 & -0.88202 & -89.60667 & 23.0 & 12.0 \\
\hline G_SC_IL-52 & 215 & -0.88027 & -89.60098 & 20.0 & 18.6 \\
\hline G_SC_IL-53 & 333 & -0.88158 & -89.5994 & 7.0 & 25.1 \\
\hline G_SC_IL-54 & 334 & -0.87902 & -89.5994 & 16.0 & 22.2 \\
\hline G_SC_IL-55 & 212 & -0.877 & -89.60005 & 30.0 & 15.7 \\
\hline G_SC_IL-57 & 210 & -0.87578 & -89.59875 & 37.0 & 13.7 \\
\hline G_E_IG-58 & 104 & -1.34412 & -89.6483 & 13.0 & 19.2 \\
\hline G_E_IG-59 & 339 & -1.34412 & -89.64923 & 14.0 & 12.9 \\
\hline G_E_IG-60 & 207 & -1.34473 & -89.6506 & 14.0 & 13.3 \\
\hline G_E_IG-61 & 110 & -1.34492 & -89.65232 & 23.0 & 20.9 \\
\hline G_E_IG-62 & 517 & -1.34523 & -89.65385 & 22.0 & 20.9 \\
\hline G_E_IG-63 & 106 & -1.34513 & -89.65605 & 19.0 & 16.1 \\
\hline G_E_IG-64 & 108 & -1.34517 & -89.65835 & 17.0 & 17.4 \\
\hline G_E_IG-65 & 107 & -1.3453 & -89.66225 & 15.0 & 13.8 \\
\hline G_E_IG-66 & 201 & -1.346 & -89.66257 & 20.0 & 9.8 \\
\hline G_E_IG-67 & 520 & -1.34748 & -89.66113 & 10.0 & 22.3 \\
\hline G_E_IG-68 & 515 & -1.34955 & -89.6619 & 5.0 & 28.7 \\
\hline G_E_IG-69 & 513 & -1.34972 & -89.65913 & 10.0 & 34.8 \\
\hline G_E_IG-70 & 519 & -1.34933 & -89.65695 & 10.0 & 18.7 \\
\hline G_E_IG-72 & 301 & -1.34808 & -89.6509 & 11.0 & 23.7 \\
\hline G_E_IG-73 & 303 & -1.34827 & -89.65002 & 8.0 & 34.6 \\
\hline G_E_IG-75 & 304 & -1.3455 & -89.6353 & 25.0 & 35.5 \\
\hline G_E_IG-76 & 226 & -1.34483 & -89.634 & 32.0 & 14.7 \\
\hline G_E_IG-79 & 302 & -1.34845 & -89.65155 & 11.0 & 29.2 \\
\hline G_E_IG-80 & 342 & -1.34845 & -89.65155 & 11.0 & 27.1 \\
\hline G_E_IG-81 & 306 & -1.36009 & -89.64194 & 7.0 & 31.7 \\
\hline G_E_IG-82 & 507 & -1.35672 & -89.64717 & 3.0 & 34.8 \\
\hline G_E_IG-83 & 307 & -1.35637 & -89.64598 & 4.0 & 26.8 \\
\hline G_E_IG-84 & 516 & -1.35585 & -89.64505 & 6.0 & 26.7 \\
\hline G_E_IG-85 & 518 & -1.3554 & -89.64285 & 10.0 & 24.2 \\
\hline G_E_IG-86 & 206 & -1.35463 & -89.64103 & 12.0 & 3.7 \\
\hline G_E_IG-87 & 209 & -1.362 & -89.63785 & 20.0 & 14.7 \\
\hline G_E_IG-88 & 216 & -1.35362 & -89.6362 & 29.0 & 23.0 \\
\hline G_F_CDD-89 & 313 & -1.35288 & -89.63477 & 37.0 & 36.1 \\
\hline G_F_CDD-92 & 213 & -1.223 & -90.4311 & 8.0 & 20.1 \\
\hline G_F_CDD-94 & 219 & -1.22025 & -90.43298 & 21.0 & 20.1 \\
\hline G_F_CDD-95 & 309 & -1.21895 & -90.43438 & 33.0 & 27.5 \\
\hline G_F_CDD-96 & 310 & -1.21708 & -90.43095 & 34.0 & 25.2 \\
\hline G_F_CDD-97 & 343 & -1.2154 & -90.42818 & 28.0 & 25.8 \\
\hline G_F_CDD-98 & 305 & -1.21863 & -90.42823 & 20.0 & 30.7 \\
\hline G_F_CDD-99 & 319 & -1.21933 & -90.4327 & 20.0 & 37.0 \\
\hline
\end{tabular}




\begin{tabular}{|c|c|c|c|c|c|}
\hline $\begin{array}{l}\text { Sample } \\
(62 \mu m-2 m m)\end{array}$ & Cluster Bin & Degree Dec & Degree Dec & Depth (m) & Mollusc (\%) \\
\hline G_F_CDD-100 & 111 & -1.21738 & -90.42682 & 21.0 & 20.5 \\
\hline G_F_CDD-101 & 101 & -1.21592 & -90.42585 & 22.0 & 23.3 \\
\hline G_F_CDD-102 & 221 & -1.2139 & -90.42593 & 31.0 & 22.0 \\
\hline G_F_CDD-103 & 222 & -1.21275 & -90.42633 & 34.0 & 24.1 \\
\hline G_F_CDD-104 & 322 & -1.21633 & -90.42385 & 20.0 & 16.8 \\
\hline G_F_CDD-105 & 112 & -1.21633 & -90.42385 & 17.0 & 15.4 \\
\hline G_F_CDD-107 & 113 & -1.21633 & -90.42385 & 10.0 & 11.8 \\
\hline G_F_CDD-109 & 118 & -1.2156 & -90.42075 & 20.0 & 12.2 \\
\hline G_F_CDD-110 & 109 & -1.21813 & -90.42002 & 28.0 & 19.6 \\
\hline G_F_CDD-111 & 341 & -1.22173 & -90.42027 & 13.0 & 18.5 \\
\hline G_F_CDD-112 & 102 & -1.22527 & -90.41932 & 23.0 & 25.3 \\
\hline G_F_CDD-113 & 114 & -1.22827 & -90.41998 & 14.0 & 25.7 \\
\hline G_F_CDD-114 & 521 & -1.23163 & -90.41862 & 11.0 & 25.3 \\
\hline G_F_CDD-115 & 105 & -1.23372 & -90.41752 & 8.0 & 16.8 \\
\hline G_F_CDD-116 & 223 & -1.23217 & -90.41633 & 14.0 & 21.5 \\
\hline G_F_CDD-117 & 214 & -1.2284 & -90.4154 & 31.0 & 19.7 \\
\hline G_I_EB-118 & 336 & -0.6428 & -91.21093 & 35.0 & 24.4 \\
\hline G_I_EB-121 & 353 & -0.65633 & -91.19768 & 18.0 & 26.1 \\
\hline G_I_EB-123 & 220 & -0.60263 & -91.08193 & 8.0 & 19.4 \\
\hline G_I_EB-124 & 347 & -0.60463 & -91.08218 & 18.0 & 31.6 \\
\hline G_I_EB-125 & 349 & -0.60748 & -91.07947 & 19.0 & 24.2 \\
\hline G_I_EB-126 & 350 & -0.60858 & -91.08378 & 20.0 & 24.5 \\
\hline G_I_EB-135 & 204 & -0.68113 & -91.18578 & 35.0 & 9.7 \\
\hline G_I_EB-139 & 351 & -0.61475 & -91.08967 & 15.0 & 23.8 \\
\hline G_I_EB-140 & 348 & -0.59633 & -91.08692 & 37.0 & 28.8 \\
\hline G_I_EB-141 & 354 & -0.59527 & -91.08527 & 14.0 & 24.0 \\
\hline G_I_EB-142 & 355 & -0.5917 & -91.09317 & 14.0 & 25.3 \\
\hline G_I_EB-143 & 327 & -0.59148 & -91.08785 & 25.0 & 33.9 \\
\hline G_I_EB-146 & 352 & -0.58715 & -91.0992 & 22.0 & 18.2 \\
\hline G_I_UB-169 & 323 & -0.41015 & -91.22785 & 10.0 & 28.6 \\
\hline G_I_UB-170 & 324 & -0.41015 & -91.22785 & 10.0 & 27.0 \\
\hline G_I_UB-171 & 325 & -0.41015 & -91.22785 & 8.0 & 31.2 \\
\hline G_I_UB-172 & 329 & -0.41015 & -91.22785 & 8.0 & 36.3 \\
\hline G_I_UB-173 & 330 & -0.41015 & -91.22785 & 7.0 & 35.0 \\
\hline
\end{tabular}

$\begin{array}{lrrrrr}\text { Sample } & \text { Cor Alg (\%) } & \text { Serpulid (\%) } & \text { Coral (\%) } & \text { Bryo (\%) } & \text { Echino (\%) } \\ (62 \mu \mathrm{m}-2 \mathrm{~mm}) & & & & & \\ \text { D_A_43 } & 6.4 & 1.4 & 54.6 & 1.8 & 2.1 \\ \text { D_B_43 } & 2.9 & 4.7 & 55.6 & 4.3 & 3.9 \\ \text { DRS } & 10.0 & 3.3 & 61.5 & 2.7 & 2.3 \\ \text { DS_2A } & 2.0 & 2.3 & 60.3 & 8.0 & 2.0 \\ \text { DS_2B } & 5.7 & 2.0 & 61.2 & 4.0 & 0.0 \\ \text { DS_7 } & 7.6 & 2.1 & 55.0 & 4.2 & 2.1\end{array}$




\begin{tabular}{|c|c|c|c|c|c|}
\hline $\begin{array}{l}\text { Sample } \\
(62 \mu \mathrm{m}-2 \mathrm{~mm})\end{array}$ & Cor Alg (\%) & Serpulid (\%) & Coral (\%) & Bryo (\%) & Echino (\%) \\
\hline DS_9 & 6.6 & 3.5 & 55.1 & 6.6 & 2.8 \\
\hline G_B-1 & 16.2 & 11.4 & 21.9 & 7.4 & 4.4 \\
\hline G_B-2 & 9.4 & 13.1 & 26.6 & 5.4 & 5.4 \\
\hline G_B-3 & 17.4 & 19.1 & 15.0 & 10.6 & 8.2 \\
\hline G_B-5 & 6.0 & 6.4 & 23.4 & 5.7 & 4.0 \\
\hline G_B-6 & 6.1 & 8.5 & 31.6 & 6.1 & 6.5 \\
\hline G_SF_N-7 & 13.5 & 5.5 & 41.5 & 3.1 & 2.4 \\
\hline G_SF_N-8 & 12.6 & 11.1 & 20.7 & 5.9 & 5.9 \\
\hline G_SF_N-9 & 8.6 & 6.9 & 32.1 & 6.9 & 5.2 \\
\hline G_SF_N10 & 5.4 & 11.6 & 37.4 & 4.8 & 2.0 \\
\hline G_SF_N-11 & 15.4 & 11.9 & 23.4 & 4.2 & 3.1 \\
\hline G_SF_N-12 & 7.9 & 9.3 & 24.8 & 5.2 & 4.1 \\
\hline G_SF_N-13 & 8.5 & 11.9 & 15.9 & 7.8 & 3.4 \\
\hline G_SC_BM-14 & 26.3 & 7.8 & 7.5 & 5.1 & 15.0 \\
\hline G_SC_BM-15 & 21.0 & 10.0 & 11.4 & 13.5 & 6.0 \\
\hline G_SC_BM-16 & 29.2 & 14.1 & 12.3 & 9.9 & 8.5 \\
\hline G_SC_BM-17 & 23.9 & 15.3 & 10.4 & 10.1 & 3.7 \\
\hline G_SC_BM-18 & 14.7 & 22.2 & 15.7 & 4.1 & 3.8 \\
\hline G_SC_BM-19 & 19.2 & 13.4 & 12.7 & 5.8 & 1.7 \\
\hline G_SC_BM-20 & 13.9 & 19.6 & 10.8 & 9.8 & 3.0 \\
\hline G_SC_BM-21 & 15.2 & 17.2 & 7.1 & 10.8 & 1.0 \\
\hline G_SC_BM-22 & 12.0 & 21.1 & 6.0 & 11.0 & 2.7 \\
\hline G_SC_BM-23 & 13.3 & 14.3 & 6.8 & 11.3 & 4.1 \\
\hline G_SC_IL-24 & 19.3 & 17.9 & 5.5 & 10.7 & 10.3 \\
\hline G_SC_IL-25 & 12.1 & 15.4 & 4.4 & 11.1 & 6.7 \\
\hline G_SC_IL-26 & 14.1 & 23.9 & 6.7 & 11.1 & 6.4 \\
\hline G_SC_IL-27 & 6.4 & 12.5 & 16.9 & 5.8 & 6.4 \\
\hline G_SC_IL-28 & 4.4 & 9.2 & 18.7 & 8.5 & 12.2 \\
\hline G_SC_IL-29 & 15.1 & 10.8 & 15.5 & 4.7 & 3.6 \\
\hline G_SC_IL-30 & 34.0 & 7.3 & 19.3 & 6.3 & 12.0 \\
\hline G_SC_IL-31 & 24.2 & 18.8 & 9.2 & 18.4 & 7.5 \\
\hline G_SC_IL-32 & 8.9 & 8.2 & 17.2 & 10.7 & 2.4 \\
\hline G_SC_IL-33 & 38.0 & 9.7 & 8.7 & 18.7 & 3.3 \\
\hline G_SC_IL-35 & 32.4 & 10.7 & 3.0 & 22.7 & 5.0 \\
\hline G_SC_IL-36 & 68.9 & 6.1 & 3.7 & 11.1 & 2.4 \\
\hline G_SC_IL-38 & 51.2 & 15.1 & 4.7 & 8.0 & 4.7 \\
\hline G_SC_IL-39 & 12.8 & 17.4 & 14.9 & 14.9 & 3.9 \\
\hline G_SC_IL-40 & 42.4 & 6.9 & 15.5 & 5.5 & 2.1 \\
\hline G_SC_IL-41 & 38.8 & 8.8 & 12.2 & 5.8 & 6.8 \\
\hline G_SC_IL-42 & 24.1 & 18.0 & 6.5 & 11.9 & 5.4 \\
\hline G_SC_IL-43 & 39.3 & 7.8 & 1.4 & 9.8 & 10.5 \\
\hline G_SC_IL-44 & 64.2 & 3.7 & 2.0 & 7.7 & 3.3 \\
\hline G_SC_IL-45 & 32.3 & 9.7 & 1.7 & 13.9 & 17.4 \\
\hline G_SC_IL-46 & 13.1 & 7.6 & 4.7 & 5.1 & 5.1 \\
\hline G_SC_IL-47 & 24.2 & 11.8 & 4.7 & 7.1 & 13.1 \\
\hline G_SC_IL-48 & 50.7 & 8.1 & 1.7 & 9.1 & 4.0 \\
\hline G_SC_IL-50 & 64.7 & 9.7 & 1.3 & 5.3 & 5.0 \\
\hline
\end{tabular}




\begin{tabular}{|c|c|c|c|c|c|}
\hline $\begin{array}{l}\text { Sample } \\
(62 \mu m-2 m m)\end{array}$ & Cor Alg (\%) & Serpulid (\%) & Coral (\%) & Bryo (\%) & Echino (\%) \\
\hline G_SC_IL-52 & 42.6 & 10.8 & 5.4 & 11.1 & 6.1 \\
\hline G_SC_IL-53 & 12.5 & 7.9 & 12.2 & 17.6 & 6.8 \\
\hline G_SC_IL-54 & 14.3 & 11.6 & 3.8 & 19.8 & 9.2 \\
\hline G_SC_IL-55 & 50.8 & 10.0 & 4.3 & 8.7 & 5.4 \\
\hline G_SC_IL-57 & 53.2 & 9.0 & 1.7 & 5.7 & 6.7 \\
\hline G_E_IG-58 & 29.2 & 8.8 & 14.6 & 13.8 & 4.2 \\
\hline G_E_IG-59 & 23.0 & 16.7 & 12.5 & 18.1 & 6.3 \\
\hline G_E_IG-60 & 51.0 & 7.3 & 4.3 & 7.7 & 13.3 \\
\hline G_E_IG-61 & 23.6 & 8.8 & 18.5 & 15.8 & 3.0 \\
\hline G_E_IG-62 & 15.5 & 5.7 & 33.3 & 14.5 & 1.7 \\
\hline G_E_IG-63 & 27.8 & 9.7 & 15.7 & 19.7 & 3.3 \\
\hline G_E_IG-64 & 21.1 & 13.1 & 26.2 & 13.4 & 2.3 \\
\hline G_E_IG-65 & 24.2 & 9.7 & 16.8 & 19.5 & 4.4 \\
\hline G_E_IG-66 & 57.3 & 6.8 & 4.1 & 13.9 & 3.1 \\
\hline G_E_IG-67 & 21.0 & 10.3 & 33.0 & 2.0 & 5.0 \\
\hline G_E_IG-68 & 9.3 & 5.7 & 48.7 & 2.7 & 1.3 \\
\hline G_E_IG-69 & 3.7 & 7.4 & 44.5 & 5.7 & 2.0 \\
\hline G_E_IG-70 & 18.3 & 8.7 & 43.3 & 4.7 & 3.3 \\
\hline G_E_IG-72 & 21.0 & 7.3 & 9.3 & 30.0 & 3.7 \\
\hline G_E_IG-73 & 10.6 & 9.2 & 11.6 & 22.9 & 2.7 \\
\hline G_E_IG-75 & 5.0 & 12.7 & 14.4 & 17.1 & 4.0 \\
\hline G_E_IG-76 & 43.1 & 7.7 & 5.0 & 22.4 & 2.7 \\
\hline G_E_IG-79 & 12.8 & 3.4 & 17.8 & 29.9 & 3.0 \\
\hline G_E_IG-80 & 18.7 & 15.1 & 9.0 & 18.1 & 5.0 \\
\hline G_E_IG-81 & 15.3 & 11.0 & 18.0 & 13.7 & 3.0 \\
\hline G_E_IG-82 & 15.4 & 3.3 & 28.4 & 10.0 & 6.0 \\
\hline G_E_IG-83 & 17.7 & 7.0 & 21.4 & 15.7 & 5.0 \\
\hline G_E_IG-84 & 14.2 & 5.4 & 29.4 & 13.5 & 6.4 \\
\hline G_E_IG-85 & 15.1 & 6.4 & 30.2 & 15.8 & 2.7 \\
\hline G_E_IG-86 & 77.3 & 3.0 & 2.0 & 8.0 & 2.7 \\
\hline G_E_IG-87 & 51.8 & 5.7 & 8.0 & 9.0 & 6.0 \\
\hline G_E_IG-88 & 44.7 & 6.7 & 6.0 & 9.0 & 3.0 \\
\hline G_F_CDD-89 & 25.4 & 11.7 & 5.0 & 11.7 & 4.0 \\
\hline G_F_CDD-92 & 52.1 & 12.2 & 3.1 & 3.5 & 2.8 \\
\hline G_F_CDD-94 & 38.5 & 16.4 & 1.0 & 12.0 & 7.0 \\
\hline G_F_CDD-95 & 26.1 & 16.6 & 3.1 & 15.6 & 3.4 \\
\hline G_F_CDD-96 & 26.8 & 13.8 & 6.4 & 14.4 & 4.0 \\
\hline G_F_CDD-97 & 19.9 & 14.3 & 9.4 & 11.8 & 3.5 \\
\hline G_F_CDD-98 & 19.0 & 5.2 & 15.5 & 10.3 & 9.7 \\
\hline G_F_CDD-99 & 18.7 & 11.3 & 8.1 & 7.0 & 2.8 \\
\hline G_F_CDD-100 & 25.6 & 5.4 & 16.8 & 12.1 & 4.0 \\
\hline G_F_CDD-101 & 27.2 & 10.5 & 10.8 & 5.9 & 5.2 \\
\hline G_F_CDD-102 & 35.3 & 6.4 & 6.1 & 14.9 & 1.4 \\
\hline G_F_CDD-103 & 37.3 & 6.1 & 6.8 & 15.3 & 4.7 \\
\hline G_F_CDD-104 & 11.1 & 8.1 & 10.8 & 8.1 & 2.0 \\
\hline G_F_CDD-105 & 28.5 & 5.4 & 34.6 & 7.7 & 0.7 \\
\hline G_F_CDD-107 & 33.0 & 5.7 & 30.6 & 9.1 & 3.7 \\
\hline
\end{tabular}




\begin{tabular}{|c|c|c|c|c|c|}
\hline $\begin{array}{l}\text { Sample } \\
(62 \mu m-2 m m)\end{array}$ & Cor Alg (\%) & Serpulid (\%) & Coral (\%) & Bryo (\%) & Echino (\%) \\
\hline G_F_CDD-109 & 32.1 & 5.7 & 22.6 & 11.8 & 5.1 \\
\hline G_F_CDD-110 & 28.0 & 8.1 & 23.6 & 5.7 & 1.4 \\
\hline G_F_CDD-111 & 12.7 & 18.5 & 17.8 & 15.1 & 2.7 \\
\hline G_F_CDD-112 & 27.0 & 10.7 & 13.0 & 8.3 & 4.7 \\
\hline G_F_CDD-113 & 34.8 & 5.7 & 22.0 & 4.7 & 4.4 \\
\hline G_F_CDD-114 & 14.2 & 9.5 & 36.5 & 8.1 & 1.0 \\
\hline G_F_CDD-115 & 31.0 & 11.7 & 15.7 & 12.8 & 3.3 \\
\hline G_F_CDD-116 & 36.6 & 10.1 & 10.7 & 9.4 & 6.4 \\
\hline G_F_CDD-117 & 46.3 & 11.7 & 7.3 & 5.7 & 2.7 \\
\hline G_I_EB-118 & 15.1 & 13.0 & 0.3 & 4.7 & 15.7 \\
\hline G_I_EB-121 & 15.3 & 26.1 & 1.0 & 5.8 & 6.4 \\
\hline G_I_EB-123 & 35.5 & 17.4 & 0.3 & 13.0 & 6.7 \\
\hline G_I_EB-124 & 24.8 & 17.3 & 0.7 & 3.4 & 7.5 \\
\hline G_I_EB-125 & 31.3 & 16.5 & 0.7 & 6.7 & 4.0 \\
\hline G_I_EB-126 & 28.2 & 20.7 & 0.0 & 4.8 & 5.8 \\
\hline G_I_EB-135 & 74.3 & 9.3 & 0.0 & 1.0 & 1.7 \\
\hline G_I_EB-139 & 17.1 & 21.8 & 0.0 & 4.1 & 5.7 \\
\hline G_I_EB-140 & 30.2 & 17.4 & 0.7 & 4.5 & 5.9 \\
\hline G_I_EB-141 & 16.4 & 22.3 & 0.7 & 9.1 & 9.8 \\
\hline G_I_EB-142 & 17.3 & 23.1 & 0.0 & 11.6 & 5.8 \\
\hline G_I_EB-143 & 10.2 & 20.5 & 0.0 & 8.5 & 8.5 \\
\hline G_I_EB-146 & 22.5 & 25.1 & 0.0 & 6.5 & 10.9 \\
\hline G_I_UB-169 & 5.1 & 8.7 & 1.1 & 1.8 & 14.1 \\
\hline G_I_UB-170 & 8.1 & 13.1 & 0.5 & 2.7 & 8.6 \\
\hline G_I_UB-171 & 7.7 & 11.9 & 0.0 & 2.7 & 10.0 \\
\hline G_I_UB-172 & 8.3 & 13.7 & 0.0 & 6.4 & 7.8 \\
\hline G_I_UB-173 & 8.3 & 17.8 & 3.2 & 3.8 & 9.6 \\
\hline
\end{tabular}

$\begin{array}{lrrrrr}\begin{array}{l}\text { Sample } \\ (62 \mu m-2 m m)\end{array} & \text { Sponge (\%) } & \text { Barnacle (\%) } & \begin{array}{l}\text { Benthic } \\ \text { Foram (\%) }\end{array} & \begin{array}{c}\text { Planktonic } \\ \text { Foram (\%) }\end{array} & \begin{array}{l}\text { Unknown } \\ (\%)\end{array} \\ \text { D_A_43 } & 0.0 & 14.2 & 0.0 & 0.0 & 0.4 \\ \text { D_B_43 } & 0.0 & 10.8 & 1.4 & 0.0 & 0.0 \\ \text { DRS } & 0.0 & 6.4 & 0.3 & 0.0 & 0.0 \\ \text { DS_2A } & 0.0 & 6.0 & 0.3 & 0.0 & 0.3 \\ \text { DS_2B } & 0.0 & 4.7 & 0.3 & 0.0 & 0.0 \\ \text { DS_7 } & 0.0 & 14.5 & 0.3 & 0.0 & 0.3 \\ \text { DS_9 } & 0.0 & 16.0 & 0.3 & 0.0 & 0.0 \\ \text { G_B-1 } & 0.0 & 4.7 & 0.0 & 0.0 & 0.7 \\ \text { G_B-2 } & 0.0 & 4.0 & 0.3 & 0.0 & 0.3 \\ \text { G_B-3 } & 0.0 & 5.5 & 0.0 & 0.0 & 0.7 \\ \text { G_B-5 } & 0.0 & 11.4 & 0.0 & 0.0 & 0.3 \\ \text { G_B-6 } & 0.0 & 8.2 & 0.0 & 0.0 & 0.7 \\ \text { G_SF_N-7 } & 0.0 & 3.1 & 0.3 & 0.0 & 0.0 \\ \text { G_SF_N-8 } & 0.0 & 0.7 & 0.4 & 0.7\end{array}$




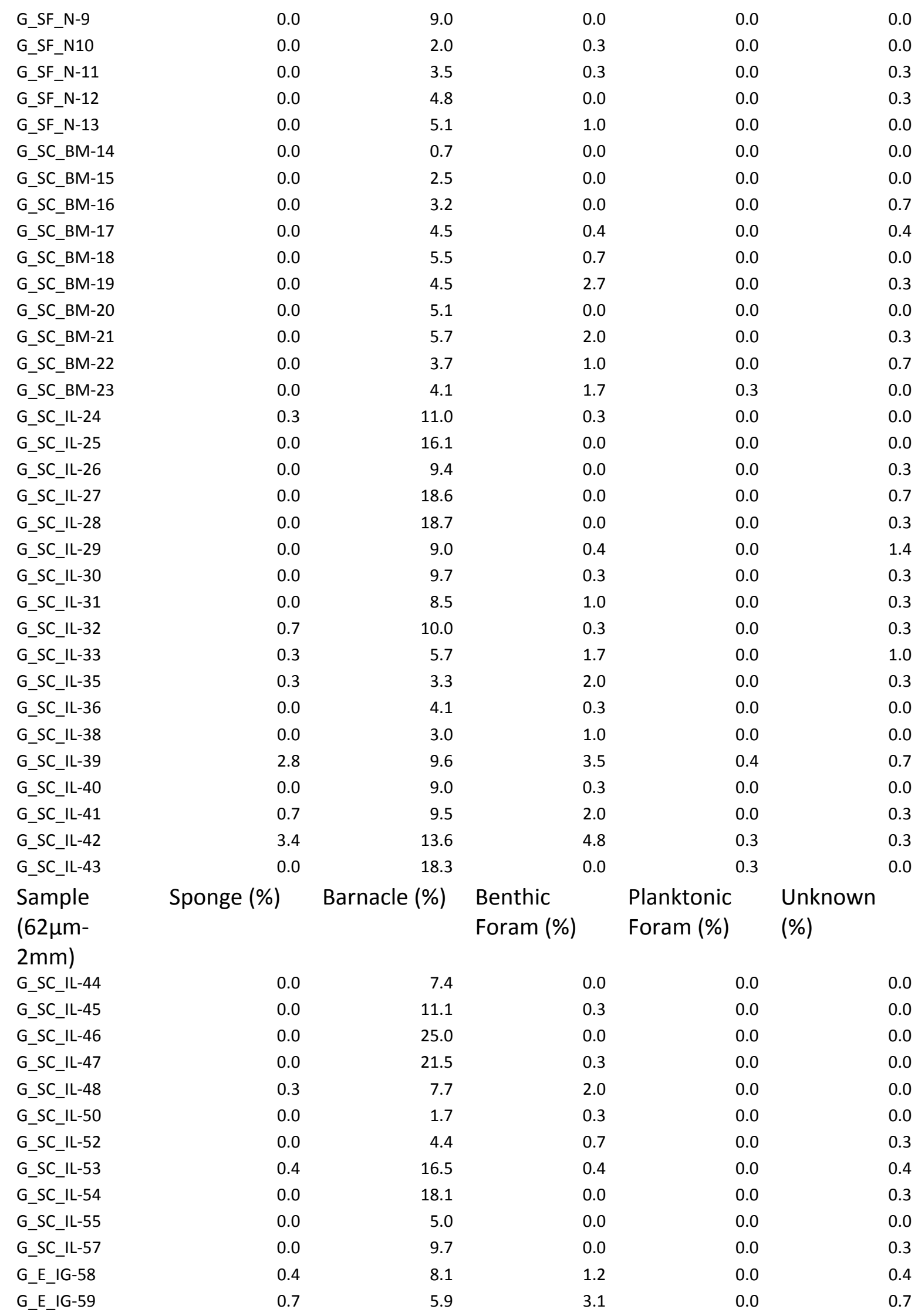




\begin{tabular}{|c|c|c|c|c|c|}
\hline $\begin{array}{l}\text { Sample } \\
(62 \mu m-2 m m)\end{array}$ & Sponge (\%) & Barnacle (\%) & $\begin{array}{l}\text { Benthic } \\
\text { Foram (\%) }\end{array}$ & $\begin{array}{l}\text { Planktonic } \\
\text { Foram (\%) }\end{array}$ & $\begin{array}{l}\text { Unknown } \\
(\%)\end{array}$ \\
\hline G_E_IG-60 & 0.7 & 2.0 & 0.0 & 0.0 & 0.3 \\
\hline G_E_IG-61 & 0.7 & 7.1 & 1.3 & 0.0 & 0.0 \\
\hline G_E_IG-62 & 0.0 & 8.1 & 0.0 & 0.0 & 0.3 \\
\hline G_E_IG-63 & 0.0 & 6.4 & 0.7 & 0.0 & 0.0 \\
\hline G_E_IG-64 & 0.0 & 5.0 & 0.0 & 0.0 & 0.0 \\
\hline G_E_IG-65 & 0.0 & 10.4 & 0.3 & 0.0 & 0.0 \\
\hline G_E_IG-66 & 0.0 & 2.0 & 2.4 & 0.3 & 0.3 \\
\hline G_E_IG-67 & 0.0 & 5.0 & 0.3 & 0.0 & 0.0 \\
\hline G_E_IG-68 & 0.0 & 2.7 & 0.7 & 0.0 & 0.3 \\
\hline G_E_IG-69 & 0.0 & 2.0 & 0.0 & 0.0 & 0.0 \\
\hline G_E_IG-70 & 0.0 & 2.0 & 0.7 & 0.0 & 0.3 \\
\hline G_E_IG-72 & 0.0 & 3.7 & 0.0 & 0.0 & 0.3 \\
\hline G_E_IG-73 & 0.0 & 5.8 & 0.3 & 0.0 & 0.3 \\
\hline G_E_IG-75 & 0.0 & 9.7 & 0.7 & 0.0 & 0.0 \\
\hline G_E_IG-76 & 0.0 & 3.7 & 0.0 & 0.0 & 0.3 \\
\hline G_E_IG-79 & 0.0 & 4.0 & 0.0 & 0.0 & 0.0 \\
\hline G_E_IG-80 & 0.0 & 6.7 & 0.0 & 0.0 & 0.0 \\
\hline G_E_IG-81 & 0.0 & 6.3 & 0.0 & 0.0 & 0.0 \\
\hline G_E_IG-82 & 0.0 & 1.3 & 0.0 & 0.0 & 0.3 \\
\hline G_E_IG-83 & 0.0 & 5.0 & 0.0 & 0.0 & 0.3 \\
\hline G_E_IG-84 & 0.0 & 3.0 & 0.0 & 0.0 & 0.7 \\
\hline G_E_IG-85 & 0.0 & 5.7 & 0.0 & 0.0 & 0.0 \\
\hline G_E_IG-86 & 0.0 & 2.3 & 0.7 & 0.0 & 0.3 \\
\hline G_E_IG-87 & 0.0 & 4.3 & 0.0 & 0.0 & 0.3 \\
\hline G_E_IG-88 & 0.0 & 7.0 & 0.3 & 0.0 & 0.3 \\
\hline G_F_CDD-89 & 0.0 & 4.3 & 0.3 & 0.0 & 0.7 \\
\hline G_F_CDD-92 & 0.0 & 3.5 & 1.0 & 0.0 & 0.3 \\
\hline G_F_CDD-94 & 0.0 & 3.3 & 0.7 & 0.0 & 0.3 \\
\hline G_F_CDD-95 & 0.0 & 3.4 & 1.4 & 0.0 & 0.3 \\
\hline G_F_CDD-96 & 0.0 & 5.4 & 3.4 & 0.0 & 0.7 \\
\hline G_F_CDD-97 & 0.0 & 11.5 & 2.8 & 0.0 & 0.7 \\
\hline G_F_CDD-98 & 0.0 & 9.7 & 0.0 & 0.0 & 0.0 \\
\hline G_F_CDD-99 & 0.0 & 9.9 & 1.1 & 0.0 & 0.4 \\
\hline G_F_CDD-100 & 0.0 & 11.4 & 1.7 & 0.0 & 0.3 \\
\hline G_F_CDD-101 & 0.0 & 14.6 & 1.4 & 0.0 & 0.3 \\
\hline G_F_CDD-102 & 0.0 & 10.8 & 2.4 & 0.0 & 0.3 \\
\hline G_F_CDD-103 & 0.0 & 3.4 & 2.0 & 0.0 & 0.0 \\
\hline G_F_CDD-104 & 0.0 & 39.1 & 0.7 & 0.0 & 0.7 \\
\hline G_F_CDD-105 & 0.0 & 4.4 & 2.7 & 0.0 & 0.3 \\
\hline G_F_CDD-107 & 0.0 & 5.1 & 1.0 & 0.0 & 0.0 \\
\hline G_F_CDD-109 & 0.0 & 8.1 & 1.4 & 0.0 & 0.7 \\
\hline G_F_CDD-110 & 0.0 & 10.8 & 1.7 & 0.0 & 0.3 \\
\hline G_F_CDD-111 & 0.0 & 11.0 & 1.7 & 0.0 & 1.0 \\
\hline G_F_CDD-112 & 0.0 & 8.7 & 1.7 & 0.0 & 0.7 \\
\hline G_F_CDD-113 & 0.0 & 1.7 & 0.3 & 0.3 & 0.3 \\
\hline G_F_CDD-114 & 0.0 & 4.4 & 0.3 & 0.0 & 0.7 \\
\hline G_F_CDD-115 & 0.0 & 4.0 & 2.2 & 0.0 & 0.4 \\
\hline
\end{tabular}




\begin{tabular}{|c|c|c|c|c|c|}
\hline $\begin{array}{l}\text { Sample } \\
(62 \mu m-2 m m)\end{array}$ & Sponge (\%) & Barnacle (\%) & $\begin{array}{l}\text { Benthic } \\
\text { Foram (\%) }\end{array}$ & $\begin{array}{l}\text { Planktonic } \\
\text { Foram (\%) }\end{array}$ & $\begin{array}{l}\text { Unknown } \\
\text { (\%) }\end{array}$ \\
\hline G_F_CDD-116 & 0.0 & 3.7 & 1.3 & 0.0 & 0.0 \\
\hline G_F_CDD-117 & 0.0 & 3.7 & 3.0 & 0.0 & 0.0 \\
\hline G_I_EB-118 & 0.0 & 23.1 & 0.0 & 0.0 & 0.3 \\
\hline G_I_EB-121 & 0.0 & 15.9 & 1.0 & 0.0 & 0.7 \\
\hline G_I_EB-123 & 0.0 & 6.4 & 0.7 & 0.0 & 0.0 \\
\hline G_I_EB-124 & 0.0 & 12.6 & 0.0 & 0.0 & 0.3 \\
\hline G_I_EB-125 & 0.0 & 13.1 & 0.3 & 0.0 & 0.7 \\
\hline G_I_EB-126 & 0.0 & 13.9 & 0.0 & 0.0 & 0.3 \\
\hline G_I_EB-135 & 0.0 & 3.7 & 0.0 & 0.0 & 0.0 \\
\hline G_I_EB-139 & 0.0 & 26.4 & 0.0 & 0.5 & 0.0 \\
\hline G_I_EB-140 & 0.0 & 11.1 & 0.3 & 0.3 & 0.0 \\
\hline G_I_EB-141 & 0.0 & 14.3 & 0.3 & 0.0 & 1.4 \\
\hline G_I_EB-142 & 0.0 & 15.5 & 0.4 & 0.0 & 0.7 \\
\hline G_I_EB-143 & 0.4 & 15.5 & 0.0 & 0.0 & 1.4 \\
\hline G_I_EB-146 & 0.0 & 16.7 & 0.0 & 0.0 & 0.0 \\
\hline G_I_UB-169 & 0.0 & 39.5 & 0.0 & 0.0 & 0.0 \\
\hline G_I_UB-170 & 0.0 & 39.6 & 0.0 & 0.0 & 0.0 \\
\hline G_I_UB-171 & 0.0 & 36.5 & 0.0 & 0.0 & 0.0 \\
\hline G_I_UB-172 & 0.0 & 25.5 & 0.5 & 0.0 & 0.5 \\
\hline G_I_UB-173 & 0.0 & 20.4 & 0.0 & 0.0 & 0.0 \\
\hline
\end{tabular}

\begin{tabular}{|c|c|c|}
\hline $\begin{array}{l}\text { Sample } \\
(62 \mu \mathrm{m}-2 \mathrm{~mm})\end{array}$ & $\begin{array}{l}\text { Crustacean } \\
(\%)\end{array}$ & Cement (\%) \\
\hline D_A_43 & 2.8 & 0.0 \\
\hline D_B_43 & 0.0 & 0.0 \\
\hline DRS & 0.7 & 0.0 \\
\hline DS_2A & 1.3 & 0.7 \\
\hline DS_2B & 1.3 & 0.0 \\
\hline DS_7 & 0.0 & 0.0 \\
\hline DS_9 & 0.0 & 0.0 \\
\hline G_B-1 & 0.0 & 0.0 \\
\hline G_B-2 & 0.0 & 0.0 \\
\hline G_B-3 & 0.0 & 0.0 \\
\hline G_B-5 & 0.0 & 0.0 \\
\hline G_B-6 & 0.0 & 0.0 \\
\hline G_SF_N-7 & 0.0 & 0.0 \\
\hline G_SF_N-8 & 1.1 & 0.0 \\
\hline G_SF_N-9 & 0.3 & 0.0 \\
\hline G_SF_N10 & 0.3 & 0.3 \\
\hline G_SF_N-11 & 0.0 & 0.0 \\
\hline G_SF_N-12 & 0.0 & 0.0 \\
\hline G_SF_N-13 & 1.0 & 0.0 \\
\hline G_SC_BM-14 & 0.7 & 0.0 \\
\hline G_SC_BM-15 & 0.4 & 0.0 \\
\hline G_SC_BM-16 & 0.0 & 0.0 \\
\hline
\end{tabular}




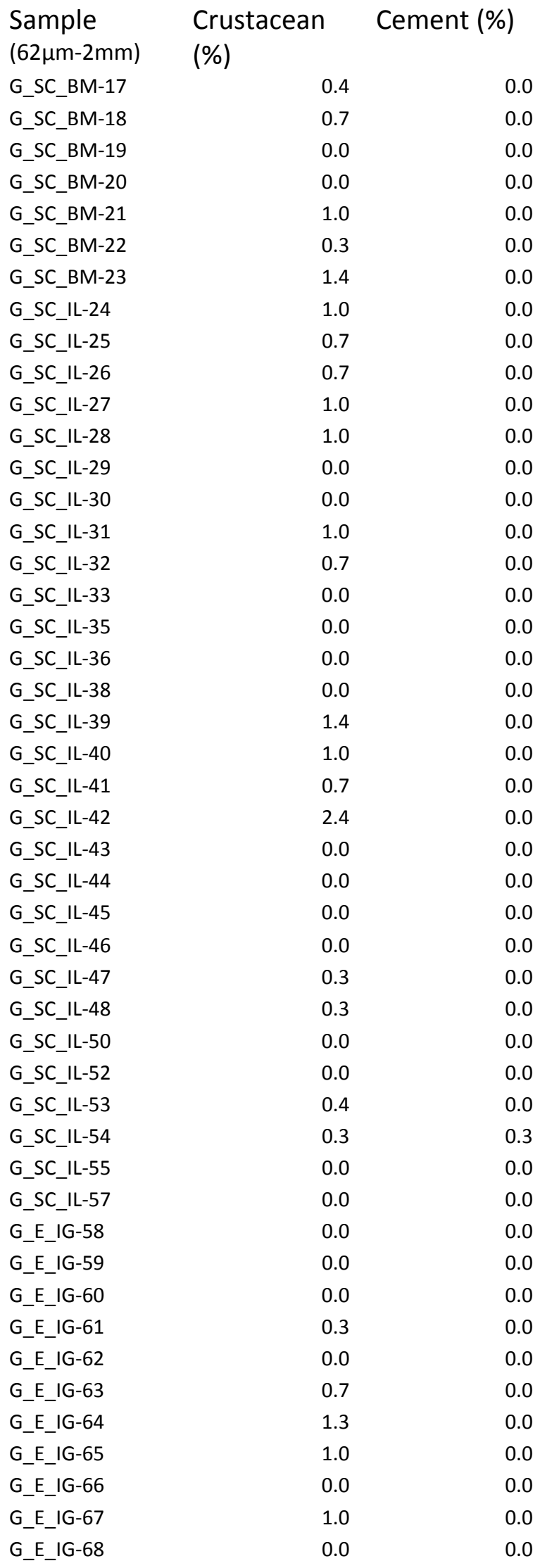




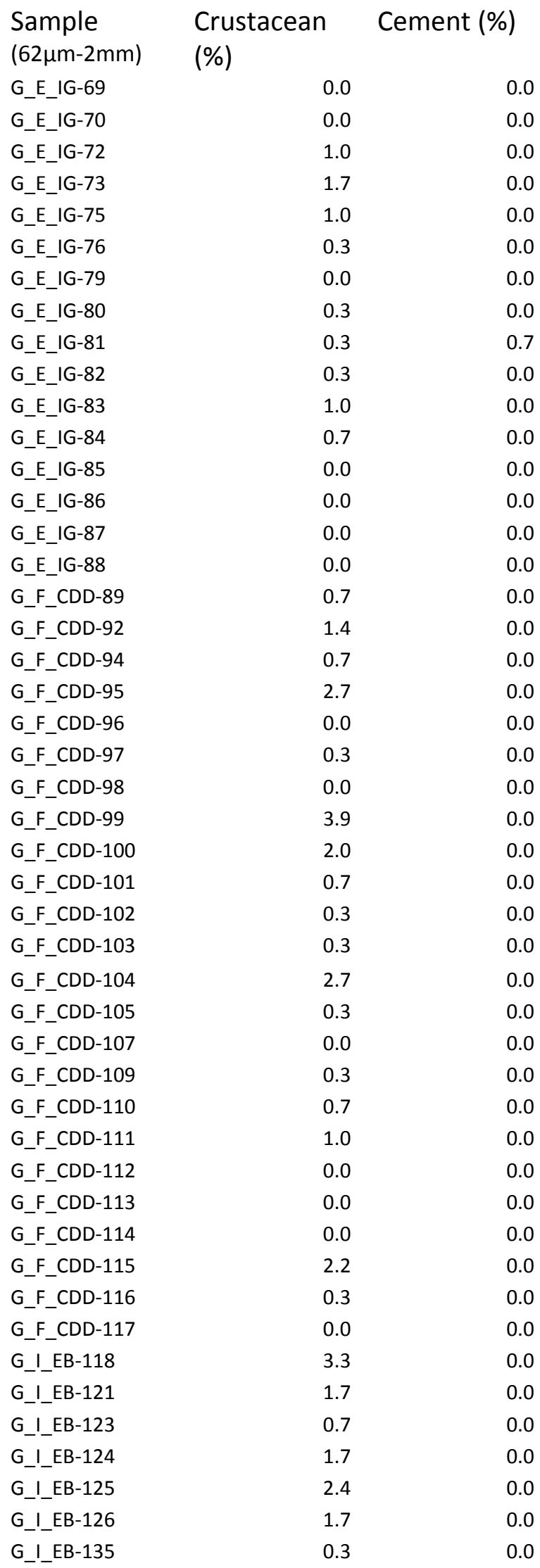




\begin{tabular}{|c|c|c|}
\hline $\begin{array}{l}\text { Sample } \\
(62 \mu m-2 m m)\end{array}$ & $\begin{array}{l}\text { Crustacean } \\
(\%)\end{array}$ & Cement (\%) \\
\hline G_I_EB-139 & 0.5 & 0.0 \\
\hline G_I_EB-140 & 0.7 & 0.0 \\
\hline G_I_EB-141 & 1.7 & 0.0 \\
\hline G_I_EB-142 & 0.4 & 0.0 \\
\hline G_I_EB-143 & 1.1 & 0.0 \\
\hline G_I_EB-146 & 0.0 & 0.0 \\
\hline G_I_UB-169 & 1.1 & 0.0 \\
\hline G_I_UB-170 & 0.5 & 0.0 \\
\hline G_I_UB-171 & 0.0 & 0.0 \\
\hline G_I_UB-172 & 1.0 & 0.0 \\
\hline G_I_UB-173 & 1.9 & 0.0 \\
\hline
\end{tabular}

Sample $(63 \mu \mathrm{m}-2 \mathrm{~mm}) \quad$ Depth $(\mathrm{m}) \quad$ Chl-a (Mean, $\left.\mathrm{mg} / \mathrm{m}^{\wedge} 3\right) \quad$ Min (Chl) Max (Chl)

$\begin{array}{lcccc}\text { D_A_43 } & 13.11 & 0.21 & 0.12 & 0.43 \\ \text { D_B_43 } & 13.11 & 0.21 & 0.12 & 0.43 \\ \text { DRS } & 12.50 & 0.21 & 0.12 & 0.43 \\ \text { DS_2A } & 13.72 & 0.21 & 0.12 & 0.43 \\ \text { DS_2B } & 13.72 & 0.21 & 0.12 & 0.43 \\ \text { DS_7 } & 13.72 & 0.21 & 0.12 & 0.43 \\ \text { DS_9 } & 13.72 & 0.21 & 0.12 & 0.43 \\ \text { G_B-1 } & 4.27 & 0.36 & 0.16 & 0.96 \\ \text { G_B-2 } & 3.66 & 0.36 & 0.16 & 0.96 \\ \text { G_B-3 } & 2.74 & 0.36 & 0.16 & 0.96 \\ \text { G_B-5 } & 3.35 & 0.36 & 0.16 & 0.96 \\ \text { G_B-6 } & 2.74 & 0.36 & 0.16 & 0.96 \\ \text { G_SF_N-7 } & 4.00 & 0.35 & 0.19 & 0.71 \\ \text { G_SF_N-8 } & 4.00 & 0.35 & 0.19 & 0.71 \\ \text { G_SF_N-9 } & 2.00 & 0.19 & 0.71 \\ \text { G_SF_N10 } & 45 & 0.19 & 0.71\end{array}$




\begin{tabular}{|c|c|c|c|c|}
\hline Sample $(63 \mu \mathrm{m}-2 \mathrm{~mm})$ & Depth (m) & Chl-a (Mean, mg/m^3) & Min (Chl) & $\operatorname{Max}(\mathrm{Chl})$ \\
\hline G_SF_N-12 & 12.00 & 0.35 & 0.19 & 0.71 \\
\hline G_SF_N-13 & 29.00 & 0.35 & 0.19 & 0.71 \\
\hline G_SC_BM-14 & 6.00 & 0.49 & 0.19 & 1.57 \\
\hline G_SC_BM-15 & 5.00 & 0.49 & 0.19 & 1.57 \\
\hline G_SC_BM-16 & 4.00 & 0.49 & 0.19 & 1.57 \\
\hline G_SC_BM-17 & 3.00 & 0.49 & 0.19 & 1.57 \\
\hline G_SC_BM-18 & 7.00 & 0.49 & 0.19 & 1.57 \\
\hline G_SC_BM-19 & 7.00 & 0.49 & 0.19 & 1.57 \\
\hline G_SC_BM-20 & 15.00 & 0.49 & 0.19 & 1.57 \\
\hline G_SC_BM-21 & 20.00 & 0.49 & 0.19 & 1.57 \\
\hline G_SC_BM-22 & 23.00 & 0.49 & 0.19 & 1.57 \\
\hline G_SC_BM-23 & 28.00 & 0.49 & 0.19 & 1.57 \\
\hline G_SC_IL-24 & 6.00 & 0.49 & 0.19 & 1.57 \\
\hline G_SC_IL-25 & 6.00 & 0.49 & 0.19 & 1.57 \\
\hline G_SC_IL-26 & 5.00 & 0.49 & 0.19 & 1.57 \\
\hline G_SC_IL-27 & 4.00 & 0.49 & 0.19 & 1.57 \\
\hline G_SC_IL-28 & 4.00 & 0.49 & 0.19 & 1.57 \\
\hline G_SC_IL-29 & 6.50 & 0.49 & 0.19 & 1.57 \\
\hline G_SC_IL-30 & 6.50 & 0.49 & 0.19 & 1.57 \\
\hline G_SC_IL-31 & 16.00 & 0.49 & 0.19 & 1.57 \\
\hline G_SC_IL-32 & 20.00 & 0.49 & 0.19 & 1.57 \\
\hline G_SC_IL-33 & 26.00 & 0.49 & 0.19 & 1.57 \\
\hline G_SC_IL-35 & 27.00 & 0.49 & 0.19 & 1.57 \\
\hline G_SC_IL-36 & 22.00 & 0.49 & 0.19 & 1.57 \\
\hline
\end{tabular}




$\begin{array}{lrrrr}\text { Sample }(63 \mu \mathrm{m}-2 \mathrm{~mm}) & \text { Depth }(\mathrm{m}) & \left.\text { Chl-a (Mean, } \mathrm{mg} / \mathrm{m}^{\wedge} 3\right) & \text { Min }(\mathrm{Chl}) & \text { Max (Chl) } \\ \text { G_SC_IL-39 } & 24.00 & 0.49 & 0.19 & 1.57 \\ \text { G_SC_IL-40 } & 24.00 & 0.49 & 0.19 & 1.57 \\ \text { G_SC_IL-41 } & 7.00 & 0.49 & 0.19 & 1.57 \\ \text { G_SC_IL-42 } & 25.00 & 0.49 & 0.19 & 1.57\end{array}$

\begin{tabular}{|c|c|c|c|c|}
\hline Sample $(63 \mu \mathrm{m}-2 \mathrm{~mm})$ & Depth (m) & Chl-a (Mean, mg/m^3) & $\operatorname{Min}(\mathrm{Chl})$ & $\operatorname{Max}(\mathrm{Chl})$ \\
\hline G_SC_IL-43 & 15.00 & 0.49 & 0.19 & 1.57 \\
\hline G_SC_IL-44 & 14.00 & 0.49 & 0.19 & 1.57 \\
\hline G_SC_IL-45 & 11.00 & 0.49 & 0.19 & 1.57 \\
\hline G_SC_IL-46 & 27.00 & 0.49 & 0.19 & 1.57 \\
\hline G_SC_IL-47 & 17.00 & 0.49 & 0.19 & 1.57 \\
\hline G_SC_IL-48 & 30.00 & 0.49 & 0.19 & 1.57 \\
\hline G_SC_IL-50 & 23.00 & 0.49 & 0.19 & 1.57 \\
\hline G_SC_IL-52 & 20.00 & 0.49 & 0.19 & 1.57 \\
\hline G_SC_IL-53 & 7.00 & 0.49 & 0.19 & 1.57 \\
\hline G_SC_IL-54 & 16.00 & 0.49 & 0.19 & 1.57 \\
\hline G_SC_IL-55 & 30.00 & 0.49 & 0.19 & 1.57 \\
\hline G_SC_IL-57 & 37.00 & 0.49 & 0.19 & 1.57 \\
\hline G_E_IG-58 & 13.00 & 0.30 & 0.18 & 0.67 \\
\hline G_E_IG-59 & 14.00 & 0.30 & 0.18 & 0.67 \\
\hline G_E_IG-60 & 14.00 & 0.30 & 0.18 & 0.67 \\
\hline G_E_IG-61 & 23.00 & 0.30 & 0.18 & 0.67 \\
\hline G_E_IG-62 & 22.00 & 0.30 & 0.18 & 0.67 \\
\hline G_E_IG-63 & 19.00 & 0.30 & 0.18 & 0.67 \\
\hline G_E_IG-64 & 17.00 & 0.30 & 0.18 & 0.67 \\
\hline G_E_IG-65 & 15.00 & 0.30 & 0.18 & 0.67 \\
\hline G_E_IG-66 & 20.00 & 0.30 & 0.18 & 0.67 \\
\hline G_E_IG-67 & 10.00 & 0.30 & 0.18 & 0.67 \\
\hline G_E_IG-68 & 5.00 & 0.30 & 0.18 & 0.67 \\
\hline G_E_IG-69 & 10.00 & 0.30 & 0.18 & 0.67 \\
\hline G_E_IG-70 & 10.00 & 0.30 & 0.18 & 0.67 \\
\hline G_E_IG-72 & 11.00 & 0.30 & 0.18 & 0.67 \\
\hline G_E_IG-73 & 8.00 & 0.30 & 0.18 & 0.67 \\
\hline G_E_IG-75 & 25.00 & 0.30 & 0.18 & 0.67 \\
\hline G_E_IG-76 & 32.00 & 0.30 & 0.18 & 0.67 \\
\hline G_E_IG-79 & 11.00 & 0.30 & 0.18 & 0.67 \\
\hline G_E_IG-80 & 11.00 & 0.30 & 0.18 & 0.67 \\
\hline G_E_IG-81 & 7.00 & 0.30 & 0.18 & 0.67 \\
\hline
\end{tabular}




\begin{tabular}{|c|c|c|c|c|}
\hline Sample $(63 \mu \mathrm{m}-2 \mathrm{~mm})$ & Depth (m) & Chl-a (Mean, $\mathrm{mg} / \mathrm{m}^{\wedge} 3$ ) & Min (Chl) & $\operatorname{Max}(\mathrm{Chl})$ \\
\hline G_E_IG-83 & 4.00 & 0.30 & 0.18 & 0.67 \\
\hline G_E_IG-84 & 6.00 & 0.30 & 0.18 & 0.67 \\
\hline G_E_IG-85 & 10.00 & 0.30 & 0.18 & 0.67 \\
\hline G_E_IG-86 & 12.00 & 0.30 & 0.18 & 0.67 \\
\hline G_E_IG-87 & 20.00 & 0.30 & 0.18 & 0.67 \\
\hline G_E_IG-88 & 29.00 & 0.30 & 0.18 & 0.67 \\
\hline G_F_CDD-89 & 37.00 & 0.30 & 0.18 & 0.67 \\
\hline G_F_CDD-92 & 8.00 & 0.33 & 0.17 & 0.64 \\
\hline G_F_CDD-94 & 21.00 & 0.33 & 0.17 & 0.64 \\
\hline G_F_CDD-95 & 33.00 & 0.33 & 0.17 & 0.64 \\
\hline G_F_CDD-96 & 34.00 & 0.33 & 0.17 & 0.64 \\
\hline G_F_CDD-97 & 28.00 & 0.33 & 0.17 & 0.64 \\
\hline G_F_CDD-98 & 20.00 & 0.33 & 0.17 & 0.64 \\
\hline G_F_CDD-99 & 20.00 & 0.33 & 0.17 & 0.64 \\
\hline G_F_CDD-100 & 21.00 & 0.33 & 0.17 & 0.64 \\
\hline G_F_CDD-101 & 22.00 & 0.33 & 0.17 & 0.64 \\
\hline G_F_CDD-102 & 31.00 & 0.33 & 0.17 & 0.64 \\
\hline G_F_CDD-103 & 34.00 & 0.33 & 0.17 & 0.64 \\
\hline G_F_CDD-104 & 20.00 & 0.33 & 0.17 & 0.64 \\
\hline G_F_CDD-105 & 17.00 & 0.33 & 0.17 & 0.64 \\
\hline G_F_CDD-107 & 10.00 & 0.33 & 0.17 & 0.64 \\
\hline G_F_CDD-109 & 20.00 & 0.33 & 0.17 & 0.64 \\
\hline G_F_CDD-110 & 28.00 & 0.33 & 0.17 & 0.64 \\
\hline G_F_CDD-111 & 13.00 & 0.33 & 0.17 & 0.64 \\
\hline G_F_CDD-112 & 23.00 & 0.33 & 0.17 & 0.64 \\
\hline G_F_CDD-113 & 14.00 & 0.33 & 0.17 & 0.64 \\
\hline G_F_CDD-114 & 11.00 & 0.33 & 0.17 & 0.64 \\
\hline G_F_CDD-115 & 8.00 & 0.33 & 0.17 & 0.64 \\
\hline G_F_CDD-116 & 14.00 & 0.33 & 0.17 & 0.64 \\
\hline G_F_CDD-117 & 31.00 & 0.33 & 0.17 & 0.64 \\
\hline G_I_EB-118 & 35.00 & 2.01 & 0.33 & 7.75 \\
\hline G_I_EB-121 & 18.00 & 2.01 & 0.33 & 7.75 \\
\hline G_I_EB-123 & 8.00 & 2.01 & 0.33 & 7.75 \\
\hline G_I_EB-124 & 18.00 & 2.01 & 0.33 & 7.75 \\
\hline G_I_EB-125 & 19.00 & 2.01 & 0.33 & 7.75 \\
\hline G_I_EB-126 & 20.00 & 2.01 & 0.33 & 7.75 \\
\hline G_I_EB-135 & 35.00 & 2.01 & 0.33 & 7.75 \\
\hline G_I_EB-139 & 15.00 & 2.01 & 0.33 & 7.75 \\
\hline G_I_EB-140 & 37.00 & 2.01 & 0.33 & 7.75 \\
\hline G_I_EB-141 & 14.00 & 2.01 & 0.33 & 7.75 \\
\hline G_I_EB-142 & 14.00 & 2.01 & 0.33 & 7.75 \\
\hline
\end{tabular}




$\begin{array}{lr}\text { G_I_EB-143 } & 25.00 \\ \text { G_I_EB-146 } & 22.00 \\ \text { G_I_UB-169 } & 10.00 \\ \text { G_I_UB-170 } & 10.00 \\ \text { G_I_UB-171 } & 8.00 \\ \text { G_I_UB-172 } & 8.00 \\ \text { G_I_UB-173 } & 7.00\end{array}$

$\begin{array}{lll}2.01 & 0.33 & 7.75 \\ 2.01 & 0.33 & 7.75 \\ 2.01 & 0.33 & 7.75 \\ 2.01 & 0.33 & 7.75 \\ 2.01 & 0.33 & 7.75 \\ 2.01 & 0.33 & 7.75 \\ 2.01 & 0.33 & 7.75\end{array}$

\begin{tabular}{|c|c|c|c|}
\hline 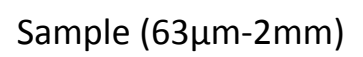 & Mean Anomaly (Chl) & SST (Mean, ${ }^{\circ} \mathrm{C}$ ) & $\operatorname{Min}(\mathrm{SST})$ \\
\hline D_A_43 & 0.04 & 25.56 & 23.30 \\
\hline D_B_43 & 0.04 & 25.56 & 23.30 \\
\hline DRS & 0.04 & 25.56 & 23.30 \\
\hline DS_2A & 0.04 & 25.56 & 23.30 \\
\hline DS_2B & 0.04 & 25.56 & 23.30 \\
\hline DS_7 & 0.04 & 25.56 & 23.30 \\
\hline DS_9 & 0.04 & 25.56 & 23.30 \\
\hline G_B-1 & 0.12 & 23.80 & 21.36 \\
\hline G_B-2 & 0.12 & 23.80 & 21.36 \\
\hline G_B-3 & 0.12 & 23.80 & 21.36 \\
\hline G_B-5 & 0.12 & 23.80 & 21.36 \\
\hline G_B-6 & 0.12 & 23.80 & 21.36 \\
\hline G_SF_N-7 & 0.10 & 23.48 & 20.67 \\
\hline G_SF_N-8 & 0.10 & 23.48 & 20.67 \\
\hline G_SF_N-9 & 0.10 & 23.48 & 20.67 \\
\hline G_SF_N10 & 0.10 & 23.48 & 20.67 \\
\hline G_SF_N-11 & 0.10 & 23.48 & 20.67 \\
\hline G_SF_N-12 & 0.10 & 23.48 & 20.67 \\
\hline G_SF_N-13 & 0.10 & 23.48 & 20.67 \\
\hline G_SC_BM-14 & 0.17 & 23.44 & 20.99 \\
\hline G_SC_BM-15 & 0.17 & 23.44 & 20.99 \\
\hline G_SC_BM-16 & 0.17 & 23.44 & 20.99 \\
\hline G_SC_BM-17 & 0.17 & 23.44 & 20.99 \\
\hline G_SC_BM-18 & 0.17 & 23.44 & 20.99 \\
\hline G_SC_BM-19 & 0.17 & 23.44 & 20.99 \\
\hline G_SC_BM-20 & 0.17 & 23.44 & 20.99 \\
\hline G_SC_BM-21 & 0.17 & 23.44 & 20.99 \\
\hline G_SC_BM-22 & 0.17 & 23.44 & 20.99 \\
\hline G_SC_BM-23 & 0.17 & 23.44 & 20.99 \\
\hline G_SC_IL-24 & 0.17 & 23.44 & 20.99 \\
\hline G SC IL-25 & 0.17 & 23.44 & 20.99 \\
\hline
\end{tabular}




\begin{tabular}{|c|c|c|c|}
\hline Sample $(63 \mu \mathrm{m}-2 \mathrm{~mm})$ & Mean Anomaly (Chl) & SST (Mean, ${ }^{\circ} \mathrm{C}$ ) & $\operatorname{Min}(\mathrm{SST})$ \\
\hline G_SC_IL-26 & 0.17 & 23.44 & 20.99 \\
\hline G_SC_IL-27 & 0.17 & 23.44 & 20.99 \\
\hline G_SC_IL-28 & 0.17 & 23.44 & 20.99 \\
\hline G_SC_IL-29 & 0.17 & 23.44 & 20.99 \\
\hline G_SC_IL-30 & 0.17 & 23.44 & 20.99 \\
\hline G_SC_IL-31 & 0.17 & 23.44 & 20.99 \\
\hline G_SC_IL-32 & 0.17 & 23.44 & 20.99 \\
\hline G_SC_IL-33 & 0.17 & 23.44 & 20.99 \\
\hline G_SC_IL-35 & 0.17 & 23.44 & 20.99 \\
\hline G_SC_IL-36 & 0.17 & 23.44 & 20.99 \\
\hline G_SC_IL-38 & 0.17 & 23.44 & 20.99 \\
\hline G_SC_IL-39 & 0.17 & 23.44 & 20.99 \\
\hline G_SC_IL-40 & 0.17 & 23.44 & 20.99 \\
\hline G_SC_IL-41 & 0.17 & 23.44 & 20.99 \\
\hline G_SC_IL-42 & 0.17 & 23.44 & 20.99 \\
\hline G_SC_IL-43 & 0.17 & 23.44 & 20.99 \\
\hline G_SC_IL-44 & 0.17 & 23.44 & 20.99 \\
\hline G_SC_IL-45 & 0.17 & 23.44 & 20.99 \\
\hline G_SC_IL-46 & 0.17 & 23.44 & 20.99 \\
\hline G_SC_IL-47 & 0.17 & 23.44 & 20.99 \\
\hline G_SC_IL-48 & 0.17 & 23.44 & 20.99 \\
\hline G_SC_IL-50 & 0.17 & 23.44 & 20.99 \\
\hline G_SC_IL-52 & 0.17 & 23.44 & 20.99 \\
\hline G_SC_IL-53 & 0.17 & 23.44 & 20.99 \\
\hline G_SC_IL-54 & 0.17 & 23.44 & 20.99 \\
\hline G_SC_IL-55 & 0.17 & 23.44 & 20.99 \\
\hline G_SC_IL-57 & 0.17 & 23.44 & 20.99 \\
\hline G_E_IG-58 & 0.11 & 23.53 & 20.75 \\
\hline G_E_IG-59 & 0.11 & 23.53 & 20.75 \\
\hline G_E_IG-60 & 0.11 & 23.53 & 20.75 \\
\hline G_E_IG-61 & 0.11 & 23.53 & 20.75 \\
\hline G_E_IG-62 & 0.11 & 23.53 & 20.75 \\
\hline G_E_IG-63 & 0.11 & 23.53 & 20.75 \\
\hline G_E_IG-64 & 0.11 & 23.53 & 20.75 \\
\hline G_E_IG-65 & 0.11 & 23.53 & 20.75 \\
\hline G_E_IG-66 & 0.11 & 23.53 & 20.75 \\
\hline G_E_IG-67 & 0.11 & 23.53 & 20.75 \\
\hline G_E_IG-68 & 0.11 & 23.53 & 20.75 \\
\hline G_E_IG-69 & 0.11 & 23.53 & 20.75 \\
\hline G_E_IG-70 & 0.11 & 23.53 & 20.75 \\
\hline G_E_IG-72 & 0.11 & 23.53 & 20.75 \\
\hline
\end{tabular}




\begin{tabular}{|c|c|c|c|}
\hline Sample $(63 \mu \mathrm{m}-2 \mathrm{~mm})$ & Mean Anomaly (Chl) & SST (Mean, ${ }^{\circ} \mathrm{C}$ ) & $\operatorname{Min}(\mathrm{SST})$ \\
\hline G_E_IG-73 & 0.11 & 23.53 & 20.75 \\
\hline G_E_IG-75 & 0.11 & 23.53 & 20.75 \\
\hline G_E_IG-76 & 0.11 & 23.53 & 20.75 \\
\hline G_E_IG-79 & 0.11 & 23.53 & 20.75 \\
\hline G_E_IG-80 & 0.11 & 23.53 & 20.75 \\
\hline G_E_IG-81 & 0.11 & 23.53 & 20.75 \\
\hline G_E_IG-82 & 0.11 & 23.53 & 20.75 \\
\hline G_E_IG-83 & 0.11 & 23.53 & 20.75 \\
\hline G_E_IG-84 & 0.11 & 23.53 & 20.75 \\
\hline G_E_IG-85 & 0.11 & 23.53 & 20.75 \\
\hline G_E_IG-86 & 0.11 & 23.53 & 20.75 \\
\hline G_E_IG-87 & 0.11 & 23.53 & 20.75 \\
\hline G_E_IG-88 & 0.11 & 23.53 & 20.75 \\
\hline G_F_CDD-89 & 0.11 & 23.53 & 20.75 \\
\hline G_F_CDD-92 & 0.07 & 23.41 & 20.29 \\
\hline G_F_CDD-94 & 0.07 & 23.41 & 20.29 \\
\hline G_F_CDD-95 & 0.07 & 23.41 & 20.29 \\
\hline G_F_CDD-96 & 0.07 & 23.41 & 20.29 \\
\hline G_F_CDD-97 & 0.07 & 23.41 & 20.29 \\
\hline G_F_CDD-98 & 0.07 & 23.41 & 20.29 \\
\hline \multicolumn{4}{|l|}{ G_F_CDD-99 } \\
\hline & 0.07 & 23.41 & 20.29 \\
\hline G_F_CDD-100 & 0.07 & 23.41 & 20.29 \\
\hline G_F_CDD-101 & 0.07 & 23.41 & 20.29 \\
\hline G_F_CDD-102 & 0.07 & 23.41 & 20.29 \\
\hline G_F_CDD-103 & 0.07 & 23.41 & 20.29 \\
\hline G_F_CDD-104 & 0.07 & 23.41 & 20.29 \\
\hline G_F_CDD-105 & 0.07 & 23.41 & 20.29 \\
\hline G_F_CDD-107 & 0.07 & 23.41 & 20.29 \\
\hline G_F_CDD-109 & 0.07 & 23.41 & 20.29 \\
\hline G_F_CDD-110 & 0.07 & 23.41 & 20.29 \\
\hline G_F_CDD-111 & 0.07 & 23.41 & 20.29 \\
\hline G_F_CDD-112 & 0.07 & 23.41 & 20.29 \\
\hline G_F_CDD-113 & 0.07 & 23.41 & 20.29 \\
\hline G_F_CDD-114 & 0.07 & 23.41 & 20.29 \\
\hline G_F_CDD-115 & 0.07 & 23.41 & 20.29 \\
\hline G_F_CDD-116 & 0.07 & 23.41 & 20.29 \\
\hline G_F_CDD-117 & 0.07 & 23.41 & 20.29 \\
\hline G_I_EB-118 & 0.52 & 22.96 & 20.26 \\
\hline G_I_EB-121 & 0.52 & 22.96 & 20.26 \\
\hline G_I_EB-123 & 0.52 & 22.96 & 20.26 \\
\hline
\end{tabular}




$\begin{array}{lrrr}\text { Sample }(63 \mu \mathrm{m}-2 \mathrm{~mm}) & \text { Mean Anomaly (Chl) } & \left.\text { SST (Mean, }{ }^{\circ} \mathrm{C}\right) & \text { Min (SST) } \\ \text { G_I_EB-125 } & 0.52 & 22.96 & 20.26 \\ \text { G_I_EB-126 } & 0.52 & 22.96 & 20.26 \\ \text { G_I_EB-135 } & 0.52 & 22.96 & 20.26 \\ \text { G_I_EB-139 } & 0.52 & 22.96 & 20.26 \\ \text { G_I_EB-140 } & 0.52 & 22.96 & 20.26 \\ \text { G_I_EB-141 } & 0.52 & 22.96 & 20.26 \\ \text { G_I_EB-142 } & 0.52 & 22.96 & 20.26 \\ \text { G_I_EB-143 } & 0.52 & 22.96 & 20.26 \\ \text { G_I_EB-146 } & 0.52 & 22.96 & 20.26 \\ \text { G_I_UB-169 } & 0.52 & 22.96 & 20.26 \\ \text { G_I_UB-170 } & 0.52 & 22.96 & 20.26 \\ \text { G_I_UB-171 } & 0.52 & 22.96 & 20.26 \\ \text { G_I_UB-172 } & 0.52 & 22.96 & 20.26 \\ \text { G_I_UB-173 } & 0.52 & 22.96 & 20.26\end{array}$

$\begin{array}{lrrr}\text { Sample }(63 \mu m-2 m m) & \text { Max (SST) } & \text { Mean Anomaly (SST) } & \text { SSS (Mean, PSU) } \\ \text { D_A_43 } & 27.90 & 3.32 & 33.54 \\ \text { D_B_43 } & 27.90 & 3.32 & 33.54 \\ \text { DRS } & 27.90 & 3.32 & 33.54 \\ \text { DS_2A } & 27.90 & 3.32 & 33.54 \\ \text { DS_2B } & 27.90 & 3.32 & 33.54 \\ \text { DS_7 } & 27.90 & 3.32 & 33.54 \\ \text { DS_9 } & 27.90 & 3.32 & 33.54 \\ \text { G_B-1 } & 27.25 & 2.77 & 34.11 \\ \text { G_B-2 } & 27.25 & 2.77 & 34.11 \\ \text { G_B-3 } & 27.25 & 2.77 & 34.11 \\ \text { G_B-5 } & 27.25 & 2.77 & 34.11 \\ \text { G_B-6 } & 27.25 & 2.77 & 34.11 \\ \text { G_SF_N-7 } & 27.01 & 2.52 & 34.25 \\ \text { G_SF_N-8 } & 27.01 & 2.52 & 34.25 \\ \text { G_SF_N-9 } & 27.01 & 2.52 & 34.25 \\ \text { G_SF_N10 } & 27.01 & 2.52 & 34.25 \\ \text { G_SF_N-11 } & 27.01 & 2.52 & 34.25 \\ \text { G_SF_N-12 } & 27.01 & 2.52 & 34.25 \\ \text { G_SF_N-13 } & 27.01 & 2.52 & 34.25 \\ \text { G_SC_BM-14 } & 26.68 & 2.73 & 34.24 \\ \text { G_SC_BM-15 } & 26.68 & 2.73 & 34.24 \\ \text { G_SC_BM-16 } & 26.68 & 2.73 & 34.24 \\ \text { G_SC_BM-17 } & 26.68 & 2.73 & 34.24 \\ \text { G_SC_BM-18 } & 26.68 & 2.73 & 34.24\end{array}$




\begin{tabular}{|c|c|c|c|}
\hline Sample $(63 \mu \mathrm{m}-2 \mathrm{~mm})$ & Max (SST) & Mean Anomaly (SST) & SSS (Mean, PSU) \\
\hline G_SC_BM-20 & 26.68 & 2.73 & 34.24 \\
\hline G_SC_BM-21 & 26.68 & 2.73 & 34.24 \\
\hline G_SC_BM-22 & 26.68 & 2.73 & 34.24 \\
\hline G_SC_BM-23 & 26.68 & 2.73 & 34.24 \\
\hline G_SC_IL-24 & 26.68 & 2.73 & 34.24 \\
\hline G_SC_IL-25 & 26.68 & 2.73 & 34.24 \\
\hline G_SC_IL-26 & 26.68 & 2.73 & 34.24 \\
\hline G_SC_IL-27 & 26.68 & 2.73 & 34.24 \\
\hline G_SC_IL-28 & 26.68 & 2.73 & 34.24 \\
\hline G_SC_IL-29 & 26.68 & 2.73 & 34.24 \\
\hline G_SC_IL-30 & 26.68 & 2.73 & 34.24 \\
\hline G_SC_IL-31 & 26.68 & 2.73 & 34.24 \\
\hline G_SC_IL-32 & 26.68 & 2.73 & 34.24 \\
\hline G_SC_IL-33 & 26.68 & 2.73 & 34.24 \\
\hline G_SC_IL-35 & 26.68 & 2.73 & 34.24 \\
\hline G_SC_IL-36 & 26.68 & 2.73 & 34.24 \\
\hline G_SC_IL-38 & 26.68 & 2.73 & 34.24 \\
\hline G_SC_IL-39 & 26.68 & 2.73 & 34.24 \\
\hline G_SC_IL-40 & 26.68 & 2.73 & 34.24 \\
\hline G_SC_IL-41 & 26.68 & 2.73 & 34.24 \\
\hline G_SC_IL-42 & 26.68 & 2.73 & 34.24 \\
\hline \multicolumn{4}{|l|}{ G_SC_IL-43 } \\
\hline & 26.68 & 2.73 & 34.24 \\
\hline G_SC_IL-44 & 26.68 & 2.73 & 34.24 \\
\hline G_SC_IL-45 & 26.68 & 2.73 & 34.24 \\
\hline G_SC_IL-46 & 26.68 & 2.73 & 34.24 \\
\hline G_SC_IL-47 & 26.68 & 2.73 & 34.24 \\
\hline G_SC_IL-48 & 26.68 & 2.73 & 34.24 \\
\hline G_SC_IL-50 & 26.68 & 2.73 & 34.24 \\
\hline G_SC_IL-52 & 26.68 & 2.73 & 34.24 \\
\hline G_SC_IL-53 & 26.68 & 2.73 & 34.24 \\
\hline G_SC_IL-54 & 26.68 & 2.73 & 34.24 \\
\hline G_SC_IL-55 & 26.68 & 2.73 & 34.24 \\
\hline G_SC_IL-57 & 26.68 & 2.73 & 34.24 \\
\hline G_E_IG-58 & 27.00 & 2.58 & 34.40 \\
\hline G_E_IG-59 & 27.00 & 2.58 & 34.40 \\
\hline G_E_IG-60 & 27.00 & 2.58 & 34.40 \\
\hline G_E_IG-61 & 27.00 & 2.58 & 34.40 \\
\hline G_E_IG-62 & 27.00 & 2.58 & 34.40 \\
\hline G_E_IG-63 & 27.00 & 2.58 & 34.40 \\
\hline G_E_IG-64 & 27.00 & 2.58 & 34.40 \\
\hline
\end{tabular}




\begin{tabular}{|c|c|c|c|}
\hline Sample (63um-2mm) & Max (SST) & Mean Anomaly (SST) & SSS (Mean, PSU) \\
\hline G_E_IG-66 & 27.00 & 2.58 & 34.40 \\
\hline G_E_IG-67 & 27.00 & 2.58 & 34.40 \\
\hline G_E_IG-68 & 27.00 & 2.58 & 34.40 \\
\hline G_E_IG-69 & 27.00 & 2.58 & 34.40 \\
\hline G_E_IG-70 & 27.00 & 2.58 & 34.40 \\
\hline G_E_IG-72 & 27.00 & 2.58 & 34.40 \\
\hline G_E_IG-73 & 27.00 & 2.58 & 34.40 \\
\hline G_E_IG-75 & 27.00 & 2.58 & 34.40 \\
\hline G_E_IG-76 & 27.00 & 2.58 & 34.40 \\
\hline G_E_IG-79 & 27.00 & 2.58 & 34.40 \\
\hline G_E_IG-80 & 27.00 & 2.58 & 34.40 \\
\hline G_E_IG-81 & 27.00 & 2.58 & 34.40 \\
\hline G_E_IG-82 & 27.00 & 2.58 & 34.40 \\
\hline G_E_IG-83 & 27.00 & 2.58 & 34.40 \\
\hline G_E_IG-84 & 27.00 & 2.58 & 34.40 \\
\hline G_E_IG-85 & 27.00 & 2.58 & 34.40 \\
\hline G_E_IG-86 & 27.00 & 2.58 & 34.40 \\
\hline G_E_IG-87 & 27.00 & 2.58 & 34.40 \\
\hline G_E_IG-88 & 27.00 & 2.58 & 34.40 \\
\hline G_F_CDD-89 & 27.00 & 2.58 & 34.40 \\
\hline G_F_CDD-92 & 26.88 & 2.04 & 34.25 \\
\hline G_F_CDD-94 & 26.88 & 2.04 & 34.25 \\
\hline G_F_CDD-95 & 26.88 & 2.04 & 34.25 \\
\hline G_F_CDD-96 & 26.88 & 2.04 & 34.25 \\
\hline G_F_CDD-97 & 26.88 & 2.04 & 34.25 \\
\hline G_F_CDD-98 & 26.88 & 2.04 & 34.25 \\
\hline \multicolumn{4}{|l|}{ G_F_CDD-99 } \\
\hline & 26.88 & 2.04 & 34.25 \\
\hline G_F_CDD-100 & 26.88 & 2.04 & 34.25 \\
\hline G_F_CDD-101 & 26.88 & 2.04 & 34.25 \\
\hline G_F_CDD-102 & 26.88 & 2.04 & 34.25 \\
\hline G_F_CDD-103 & 26.88 & 2.04 & 34.25 \\
\hline G_F_CDD-104 & 26.88 & 2.04 & 34.25 \\
\hline G_F_CDD-105 & 26.88 & 2.04 & 34.25 \\
\hline G_F_CDD-107 & 26.88 & 2.04 & 34.25 \\
\hline G_F_CDD-109 & 26.88 & 2.04 & 34.25 \\
\hline G_F_CDD-110 & 26.88 & 2.04 & 34.25 \\
\hline G_F_CDD-111 & 26.88 & 2.04 & 34.25 \\
\hline G_F_CDD-112 & 26.88 & 2.04 & 34.25 \\
\hline G_F_CDD-113 & 26.88 & 2.04 & 34.25 \\
\hline G_F_CDD-114 & 26.88 & 2.04 & 34.25 \\
\hline
\end{tabular}




\begin{tabular}{|c|c|c|c|}
\hline Sample $(63 \mu \mathrm{m}-2 \mathrm{~mm})$ & $\operatorname{Max}(\mathrm{SST})$ & Mean Anomaly (SST) & SSS (Mean, PSU) \\
\hline G_F_CDD-116 & 26.88 & 2.04 & 34.25 \\
\hline G_F_CDD-117 & 26.88 & 2.04 & 34.25 \\
\hline G_I_EB-118 & 26.47 & 2.17 & 34.23 \\
\hline G_I_EB-121 & 26.47 & 2.17 & 34.23 \\
\hline G_I_EB-123 & 26.47 & 2.17 & 34.23 \\
\hline G_I_EB-124 & 26.47 & 2.17 & 34.23 \\
\hline G_I_EB-125 & 26.47 & 2.17 & 34.23 \\
\hline G_I_EB-126 & 26.47 & 2.17 & 34.23 \\
\hline G_I_EB-135 & 26.47 & 2.17 & 34.23 \\
\hline G_I_EB-139 & 26.47 & 2.17 & 34.23 \\
\hline G_I_EB-140 & 26.47 & 2.17 & 34.23 \\
\hline G_I_EB-141 & 26.47 & 2.17 & 34.23 \\
\hline G_I_EB-142 & 26.47 & 2.17 & 34.23 \\
\hline G_I_EB-143 & 26.47 & 2.17 & 34.23 \\
\hline G_I_EB-146 & 26.47 & 2.17 & 34.23 \\
\hline G_I_UB-169 & 26.47 & 2.17 & 34.23 \\
\hline G_I_UB-170 & 26.47 & 2.17 & 34.23 \\
\hline G_I_UB-171 & 26.47 & 2.17 & 34.23 \\
\hline G_I_UB-172 & 26.47 & 2.17 & 34.23 \\
\hline G_I_UB-173 & 26.47 & 2.17 & 34.23 \\
\hline
\end{tabular}

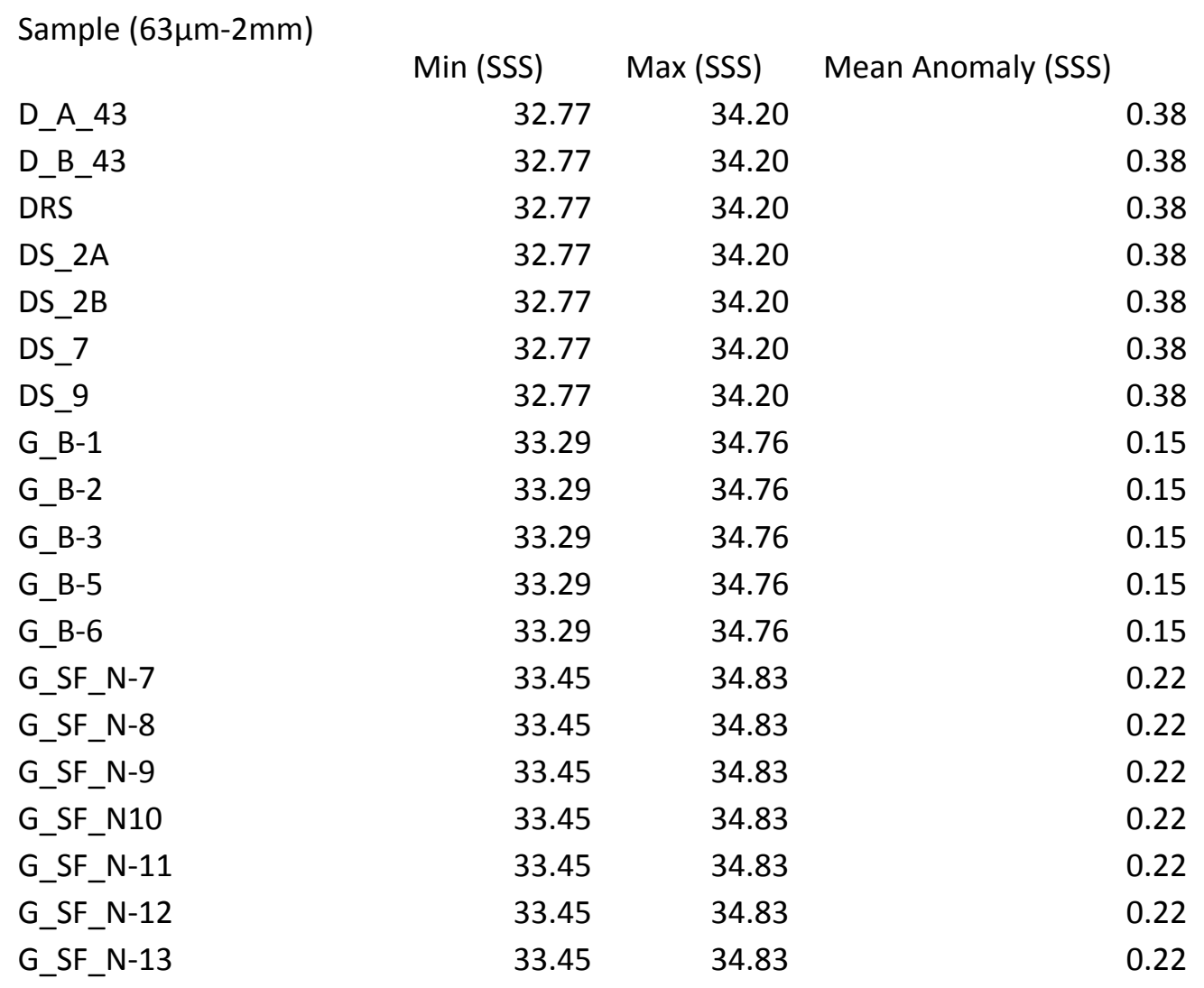




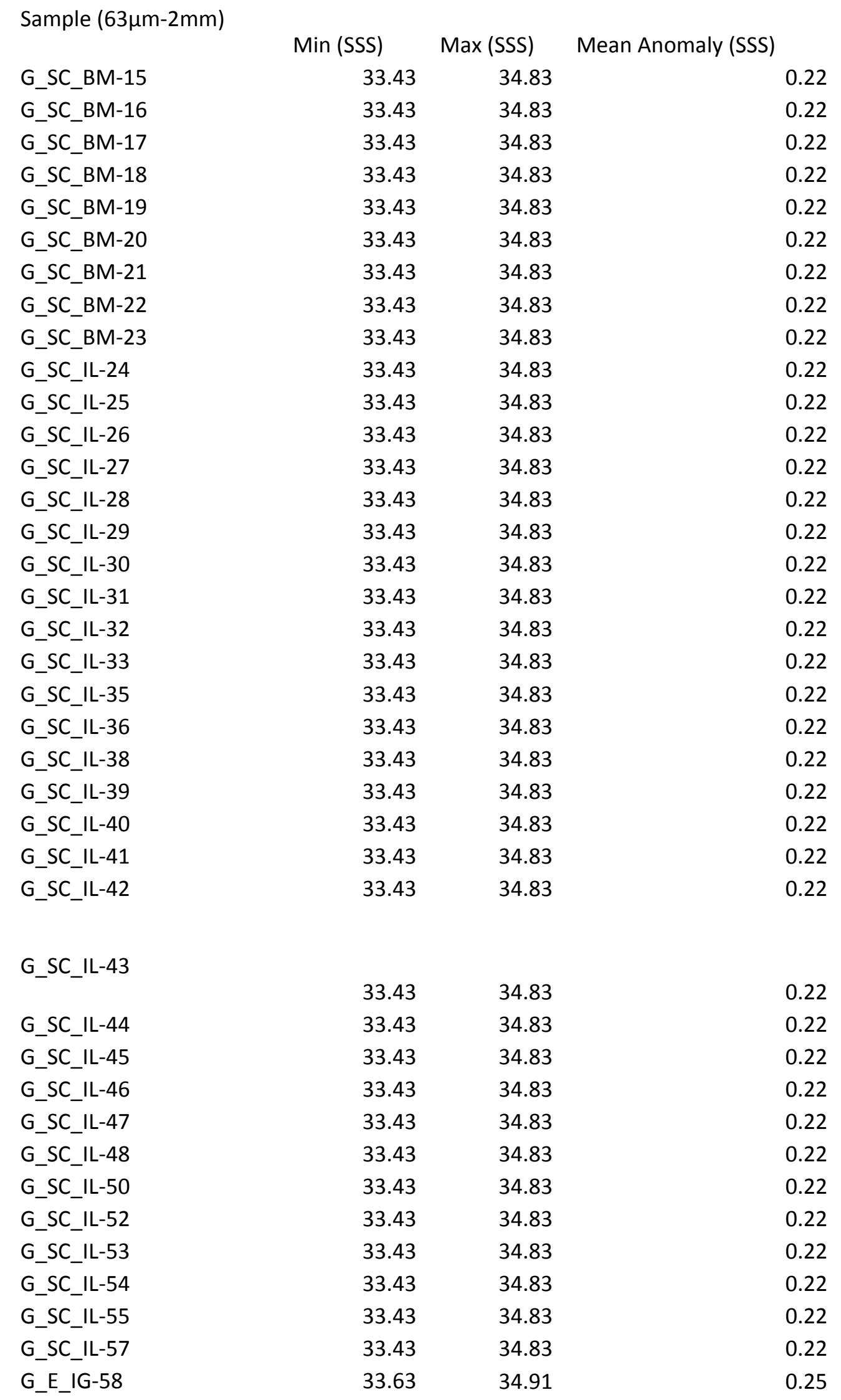




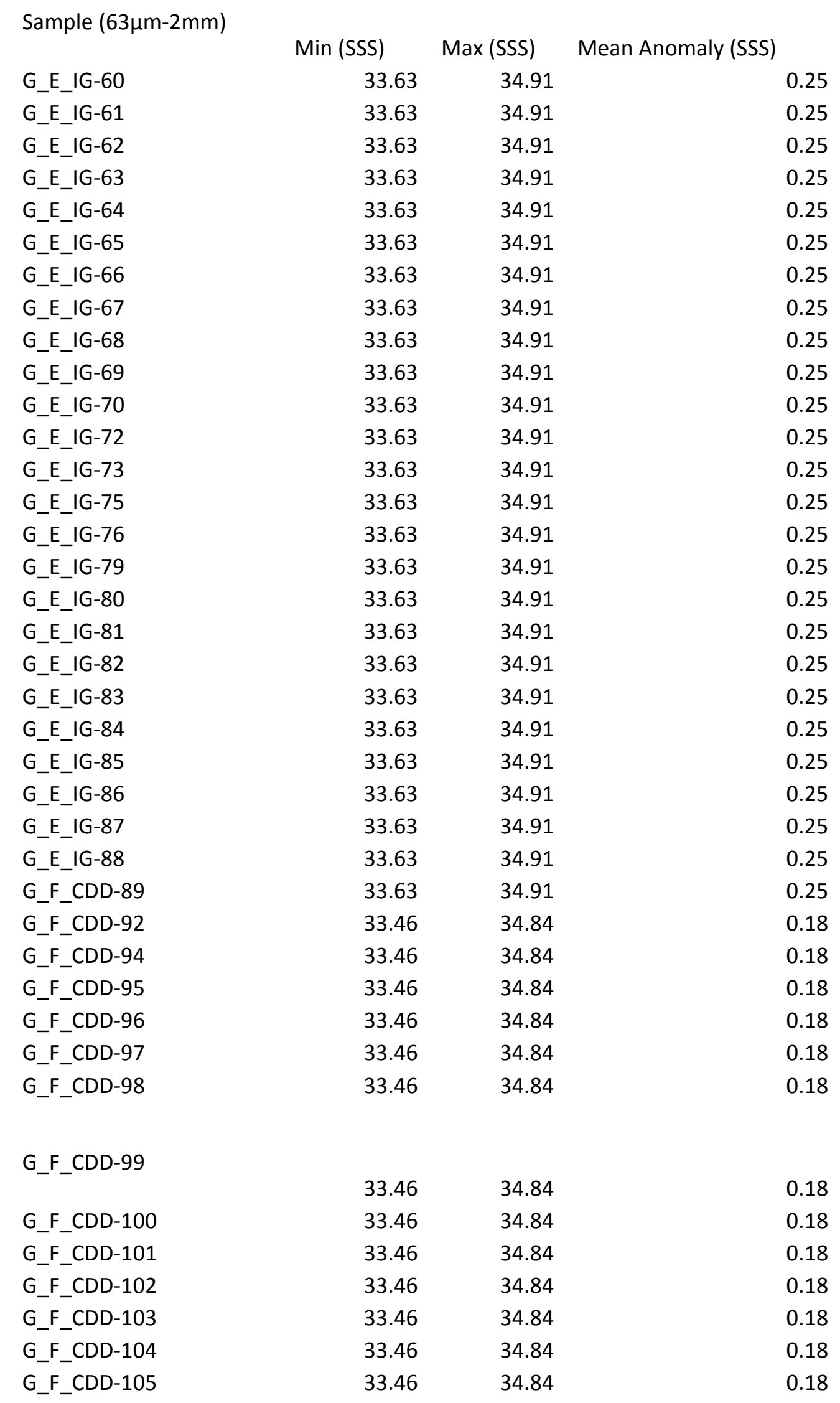




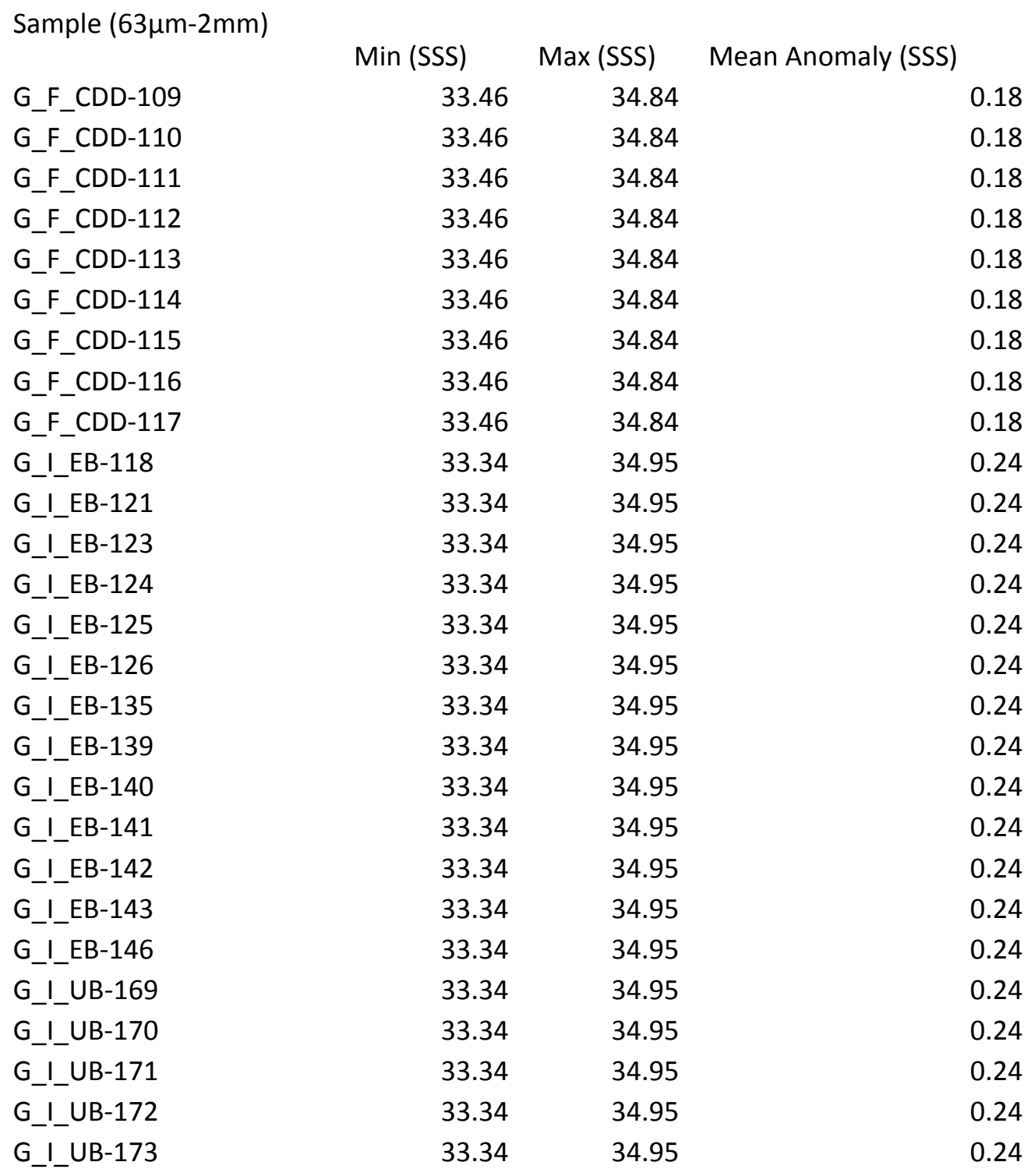

Sample $(63 \mu \mathrm{m}-2 \mathrm{~mm}) \quad$ Mean $\mathrm{pH}$ (Total Scale)

D_A_43

8.07

D_B_43

8.07

DRS

8.07

DS_2A

8.07

DS_2B

8.07

DS_7

8.07

DS_9

8.07

G_B-1

7.94

G_B-2

7.94

G_B-3

7.94 


\begin{tabular}{|c|c|}
\hline Sample $(63 \mu \mathrm{m}-2 \mathrm{~mm})$ & Mean pH (Total Scale) \\
\hline G_B-6 & 7.94 \\
\hline G_SF_N-7 & 7.97 \\
\hline G_SF_N-8 & 7.97 \\
\hline G_SF_N-9 & 7.97 \\
\hline G_SF_N10 & 7.97 \\
\hline G_SF_N-11 & 7.97 \\
\hline G_SF_N-12 & 7.97 \\
\hline G_SF_N-13 & 7.97 \\
\hline G_SC_BM-14 & 7.90 \\
\hline G_SC_BM-15 & 7.90 \\
\hline G_SC_BM-16 & 7.90 \\
\hline G_SC_BM-17 & 7.90 \\
\hline G_SC_BM-18 & 7.90 \\
\hline G_SC_BM-19 & 7.90 \\
\hline G_SC_BM-20 & 7.90 \\
\hline G_SC_BM-21 & 7.90 \\
\hline G_SC_BM-22 & 7.90 \\
\hline G_SC_BM-23 & 7.90 \\
\hline G_SC_IL-24 & 7.90 \\
\hline G_SC_IL-25 & 7.90 \\
\hline G_SC_IL-26 & 7.90 \\
\hline G_SC_IL-27 & 7.90 \\
\hline G_SC_IL-28 & 7.90 \\
\hline G_SC_IL-29 & 7.90 \\
\hline G_SC_IL-30 & 7.90 \\
\hline G_SC_IL-31 & 7.90 \\
\hline G_SC_IL-32 & 7.90 \\
\hline G_SC_IL-33 & 7.90 \\
\hline G_SC_IL-35 & 7.90 \\
\hline G_SC_IL-36 & 7.90 \\
\hline G_SC_IL-38 & 7.90 \\
\hline G_SC_IL-39 & 7.90 \\
\hline G_SC_IL-40 & 7.90 \\
\hline G_SC_IL-41 & 7.90 \\
\hline G_SC_IL-42 & 7.90 \\
\hline Sample $(63 \mu \mathrm{m}-2 \mathrm{~mm})$ & Mean pH (Total Scale) \\
\hline G_SC_IL-43 & 7.90 \\
\hline G_SC_IL-44 & 7.90 \\
\hline G_SC_IL-45 & 7.90 \\
\hline G_SC_IL-46 & 7.90 \\
\hline G_SC_IL-47 & 7.90 \\
\hline G_SC_IL-48 & 7.90 \\
\hline
\end{tabular}




\begin{tabular}{|c|c|}
\hline Sample (63um-2mm) & Mean pH (Total Scale) \\
\hline G_SC_IL-52 & 7.90 \\
\hline G_SC_IL-53 & 7.90 \\
\hline G_SC_IL-54 & 7.90 \\
\hline G_SC_IL-55 & 7.90 \\
\hline G_SC_IL-57 & 7.90 \\
\hline G_E_IG-58 & 8.03 \\
\hline G_E_IG-59 & 8.03 \\
\hline G_E_IG-60 & 8.03 \\
\hline G_E_IG-61 & 8.03 \\
\hline G_E_IG-62 & 8.03 \\
\hline G_E_IG-63 & 8.03 \\
\hline G_E_IG-64 & 8.03 \\
\hline G_E_IG-65 & 8.03 \\
\hline G_E_IG-66 & 8.03 \\
\hline G_E_IG-67 & 8.03 \\
\hline G_E_IG-68 & 8.03 \\
\hline G_E_IG-69 & 8.03 \\
\hline G_E_IG-70 & 8.03 \\
\hline G_E_IG-72 & 8.03 \\
\hline G_E_IG-73 & 8.03 \\
\hline G_E_IG-75 & 8.03 \\
\hline G_E_IG-76 & 8.03 \\
\hline G_E_IG-79 & 8.03 \\
\hline G_E_IG-80 & 8.03 \\
\hline G_E_IG-81 & 8.03 \\
\hline G_E_IG-82 & 8.03 \\
\hline G_E_IG-83 & 8.03 \\
\hline G_E_IG-84 & 8.03 \\
\hline G_E_IG-85 & 8.03 \\
\hline G_E_IG-86 & 8.03 \\
\hline G_E_IG-87 & 8.03 \\
\hline G_E_IG-88 & 8.03 \\
\hline G_F_CDD-89 & 8.03 \\
\hline G_F_CDD-92 & 7.91 \\
\hline G_F_CDD-94 & 7.91 \\
\hline G_F_CDD-95 & 7.91 \\
\hline G_F_CDD-96 & 7.91 \\
\hline G_F_CDD-97 & 7.91 \\
\hline G_F_CDD-98 & 7.91 \\
\hline Sample $(63 \mu \mathrm{m}-2 \mathrm{~mm})$ & Mean pH (Total Scale) \\
\hline G_F_CDD-99 & 7.91 \\
\hline G_F_CDD-100 & 7.91 \\
\hline
\end{tabular}




$\begin{array}{ll}\text { Sample }(63 \mu m-2 m m) & \text { Mean pH (Total Scale) } \\ \text { G_F_CDD-102 } & 7.91 \\ \text { G_F_CDD-103 } & 7.91 \\ \text { G_F_CDD-104 } & 7.91 \\ \text { G_F_CDD-105 } & 7.91 \\ \text { G_F_CDD-107 } & 7.91 \\ \text { G_F_CDD-109 } & 7.91 \\ \text { G_F_CDD-110 } & 7.91 \\ \text { G_F_CDD-111 } & 7.91 \\ \text { G_F_CDD-112 } & 7.91 \\ \text { G_F_CDD-113 } & 7.91 \\ \text { G_F_CDD-114 } & 7.91 \\ \text { G_F_CDD-115 } & 7.91 \\ \text { G_F_CDD-116 } & 7.91 \\ \text { G_F_CDD-117 } & 7.91 \\ \text { G_I_EB-118 } & 7.88 \\ \text { G_I_EB-121 } & 7.88 \\ \text { G_I_EB-123 } & 7.88 \\ \text { G_I_EB-124 } & 7.88 \\ \text { G_I_EB-125 } & 7.88 \\ \text { G_I_EB-126 } & 7.88 \\ \text { G_I_EB-135 } & 7.88 \\ \text { G_I_EB-139 } & 7.88 \\ \text { G_I_EB-140 } & 7.88 \\ \text { G_I_EB-141 } & 7.88 \\ \text { G_I_EB-142 } & 7.88 \\ \text { G_I_EB-143 } & 7.88 \\ \text { G_I_EB-146 } & 7.88 \\ \text { G_I_UB-169 } & 7.88 \\ \text { G_I_UB-170 } & 7.88 \\ \text { G_I_UB-171 } & 7.88 \\ \text { G_I_UB-172 } & \\ \text { G_I_UB-173 } & \end{array}$




\section{Table DR3.}

\begin{tabular}{|c|c|c|c|c|c|c|c|c|c|c|}
\hline Cluster Group & Mollusk \% & Coralline Algae \% & Coral \% & Serpulid \% & Bryozoan \% & Echinoderm \% & Sponge \% & acle $\%$ & $\mathrm{am} \%$ & er $\%$ \\
\hline GAL-I & 15.12 & 5.89 & 57.62 & 2.76 & 4.51 & 2.18 & 0.00 & 10.36 & 0.45 & 1.13 \\
\hline GAL-II & 32.35 & 11.68 & 30.67 & 8.49 & 7.14 & 3.73 & 0.03 & 5.04 & 0.26 & 0.61 \\
\hline GAL-III & 28.47 & 16.79 & 7.75 & 14.80 & 10.64 & 6.31 & 0.15 & 13.06 & 0.68 & 1.35 \\
\hline GAL-IV & 17.91 & 29.86 & 18.94 & 8.67 & 10.45 & 4.39 & 0.10 & 7.61 & 1.12 & 0.93 \\
\hline GAL-V & 15.33 & 48.82 & 4.02 & 9.12 & 10.64 & 5.30 & 0.06 & 5.43 & 0.88 & 0.40 \\
\hline GOC-A & 40.65 & 7.99 & 26.50 & 1.05 & 3.96 & 6.46 & 0.21 & 4.49 & 4.47 & 4.22 \\
\hline GOC-B & 17.45 & 10.80 & 0.17 & 4.05 & 46.40 & 8.57 & 0.09 & 8.68 & 1.22 & 2.57 \\
\hline GOC-C & 30.36 & 10.50 & 6.12 & 3.30 & 14.03 & 10.93 & 0.61 & 12.23 & 5.05 & 2.41 \\
\hline GOC-D & 22.51 & 34.24 & 6.63 & 2.82 & 9.06 & 8.52 & 0.43 & 3.00 & 6.74 & 6.05 \\
\hline GOC-E & 15.28 & 55.38 & 4.14 & 4.43 & 7.26 & 5.58 & 0.04 & 1.67 & 2.04 & 4.18 \\
\hline
\end{tabular}

